\title{
DNA hydroxymethylation reveals transcription regulation networks and prognostic signatures in multiple myeloma \\ Running Title: DNA Hydroxymethylation in multiple myeloma
}

\author{
Jean-Baptiste Alberge ${ }^{1}$, Florence Magrangeas ${ }^{1,2}$, Mirko Wagner $^{3}$, Soline Denié ${ }^{1}$, Catherine

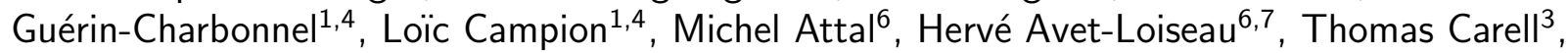 \\ Philippe Moreau ${ }^{1,2}$, Stéphane Minvielle ${ }^{1,2,{ }^{*}}$, and Aurélien A. Sérandour ${ }^{1,5, *}$ \\ ${ }^{1}$ CRCINA, INSERM, CNRS, Université d'Angers, Université de Nantes, Nantes, France \\ ${ }^{2}$ Hematology Department, University Hospital, Nantes, France \\ ${ }^{3}$ Ludwig-Maximilians-Universität München, Munich, Germany \\ ${ }^{4}$ Institut de cancérologie de l'Ouest site René-Gauducheau, Saint-Herblain, France \\ ${ }^{5}$ Ecole Centrale de Nantes, Nantes, France \\ ${ }^{6}$ Hematology Department, University Hospital, Toulouse, France \\ ${ }^{7}$ Myeloma Genomics LabIUCT-Oncopole, CRCT, INSERM, Universite Paul Sabatier, Toulouse, France \\ ${ }^{*}$ joint last authors and corresponding authors: aurelien.serandour@ec-nantes.fr stephane.minvielle@chu-nantes.fr
}

\section{Key Points :}

- $5 \mathrm{hmC}$ is globally depleted in MM and even more in advanced stages of the disease.

- $5 \mathrm{hmC}$ is locally detected at transcriptionally active regions of MM where its presence can be associated with survival.

\begin{abstract}
Multiple myeloma $(\mathrm{MM})$ is a plasma cell malignancy that remains challenging to cure despite a substantially improving median survival. During the last decade, DNA copy number variation and gene expression studies have described the pathology and its heterogeneity among patients. Epigenetic modifications play important roles in $\mathrm{MM}$, but they are rarely associated with clinical aspects of the disease. In this epigenomics study, we produced quantifications of genomic 5-methylcytosine $(5 \mathrm{mC})$ and of 5-hydroxymethylcytosine $(5 \mathrm{hmC})$ as well as genome-wide maps of hydroxymethylation to analyse myeloma cells taken from a cohort of 40 newly diagnosed and homogeneously treated patients. We found $5 \mathrm{hmC}$ to be globally depleted in MM compared to normal plasma cells, as well as being reduced in advanced clinical stages of the disease. From the hydroxymethylome data, we observed that remaining $5 \mathrm{hmC}$ is organised in large peak clusters and is associated with well-known disease-related genes. Based on their signal correlation, these $5 \mathrm{hmC}$ peak clusters can be gathered in 2 regulation networks involving core transcription factors such as IRF4, MYC, PRDM1 and TCF3. By performing paired hydroxymethylomes at diagnosis and at relapse, we found the disease progression to be heterogeneous and patient-specific. We found that the location of $5 \mathrm{hmC}$ at tumor suppressor TP53INP1 is associated with better outcome while global high level of $5 \mathrm{hmC}$ tends to be associated with better overall survival. Together, our study suggests that $5 \mathrm{hmC}$ provides new biological insights of the disease severity and progression, and can be used for retrospective studies.
\end{abstract}

\section{Correspondence:}

Aurélien Sérandour, ICO LABcT, Boulevard Jacques Monod, 44805 Saint Herblain, +33 (0)2 28080371

Stéphane Minvielle, IRS-UN, 8 quai Moncousu, 44000 Nantes, +33 (0)2 28080370

Words: 4,012, Figures: 5, Tables: 6, References: 60 


\section{Introduction}

Multiple Myeloma (MM) is a plasma cell (PC) neoplasm with an incidence rate of 5/100,000 in Europe and accounts for approximatively 1 percent of all cancers. Median survival of patients has greatly improved in the last decade $e^{[1]}$ with the use of novel strategies such as autologous stem cell transplantation and new sets of drugs: immunomodulators, proteasome inhibitors, histone deacetylase (HDAC) inhibitors and monoclonal antibodies.리 Yet treatment remains challenging as nearly all patients ultimately relapse with the emergence of a resistant subpopulation of malignant plasma cells. Malignant clones show an heterogeneous range of mutations and chromosomal abnormalities along with heterogeneous chromatin and epigenetic dysregulations at diagnosis and at relapse that affects biological pathways (MAPK, NF- $\kappa$ B, DNA-repair) $\frac{B}{3}$

Genomic and transcriptomic studies have allowed a better understanding of the disease and identified key transcription factors involved such as IRF4, MYC, PRDM1 and XBP1 ${ }^{[3.5]}$ Recent epigenomics technologies can help to deepen our knowledge of the transcriptional programs shaping MM. However epigenomics analysis through histone marks profiling with chromatin immunoprecipitation (ChIP-seq) or open chromatin mapping with Assay for Transposase-Accessible Chromatin (ATAC-seq) can be hard to set up on a cohort with limited material over a long range of time and thus give limited insights on the disease establishment and relapse. Studying DNA epigenetics marks is more adapted to this challenge.

Agirre and colleagues ${ }^{\sqrt{6}}$ described DNA methylation in an important number of MM samples. They identified very heterogeneous levels of methylation from one patient to another. They found that despite a global hypomethylation, local and extensive hypermethylation is present in $\mathrm{MM}$ at intronic enhancer regions that are associated with stem cell development.

Chatonnet and colleagues ${ }^{7}$ recently identified several hydroxymethylated $\mathrm{CpGs}$ in MM samples with limited association to disease severity or prognosis, and to our knowledge, the genome-wide mapping of $5 \mathrm{hmC}$ (hydroxymethylome) has never been studied in a well-established cohort.

Oxydative states of 5-methylated Cytosine $(5 \mathrm{mC})$ on genomic DNA have been identified a decade agd 8 10 : $5 \mathrm{hmC}, 5 \mathrm{fC}$ and $5 \mathrm{caC}$. The TET proteins TET $1 / 2 / 3$ are responsible for the $5 \mathrm{mC}$ oxidation. $5 \mathrm{fC}$ and $5 \mathrm{caC}$ are almost undetectable in genomic DNA unless the glycosylase TDG gene is knocked-out ${ }^{1112}$, whereas $5 \mathrm{hmC}$ can be found in all cell types at various levels $\sqrt{13}, 15] .5 \mathrm{hmC}$ is believed to be a DNA demethylation intermediate in a process involving TET proteins, TDG and the Base Excision Repair system.16] However $5 \mathrm{hmC}$ is also shown as a stable DNA modification 1718 Despite this discrepancy, $5 \mathrm{hmC}$ is commonly accepted as a DNA mark associated with active chromatin 1921 and is a powerful way to identify active genomic regions associated with a disease directly from genomic DNA or more recently from circulating DNA.22 23

In this study, we characterized the $5 \mathrm{hmC}$ signal genome-wide on plasma cell DNA from 40 patients newly diagnosed with MM between 2010-2012 and representative of the main molecular subtypes, including 4 paired relapse samples, and of the plasma cells of 5 control individuals.

\section{Methods}

\section{Normal plasma cells purification}

After informed consent, the femoral canal of individuals with isolated hip osteoarthritis who were otherwise healthy was probed with a metal suction device following femoral neck removal. Bone marrow cells were suctioned into a tube that contained EDTA, placed on ice and immediately transported to our laboratory. BMMCs were purified by Ficoll. Normal plasma cells were FACS-sorted using a BD FACSAria III as CD38/CD138 positive and CD3/CD13/CD33 negative (antibodies from Becton Dickinson, ref. 345807, 555332, 555394, 555450 and 562935).

\section{Myeloma cells purification}

Bone marrow biopsies were realized on MM newly diagnosed patients from cohort IFM-DFCI 2009 (Intergroupe Francophone du Myelome - Dana Farber Cancer Institute) [24.25] All patients signed an informed consent form approved by the Toulouse Ethics Committee. All included patients were newly diagnosed with symptomatic MM based on International Myeloma Working Group 2003 Diagnostic Criteria.24] All the samples were collected in France and processed at the University Hospital of Nantes. Bone Marrow Mononuclear Cells (BMMCs) were purified by Ficoll. Plasma cells were purified with anti-CD138 beads (Robosep platform, StemCell Technologies) and the CD138-positive percentage of cells was checked by immunofluorescence microscopy.

\section{Genomic DNA extraction}

Genomic DNA was extracted with Qiagen Allprep DNA/RNA Mini Kit (ref. 80204). DNA samples were dosed by DNA HS QuBit and the absence of contaminant RNA checked by RNA HS Qubit. 


\section{Patients selection for this study}

In this study, we have selected 40 patient samples with: low level or absence of RNA in DNA samples, low level of rRNA in RNA-seq data, enough DNA material available (at least $1 \mathrm{ug}$ ) and high percentage of CD138+ cells (98\% in average in this study).

\section{Digest of genomic DNA and subsequent LC-MS analysis}

The genomic levels of methylcytosine $(\mathrm{mC})$ and hydroxymethyl-cytosine $(\mathrm{hmC})$ were quantified using a mass spectrometry-based stable isotope-dilution method.26 For each LC-MS-measurement (technical replicate), 70 ng of genomic DNA (gDNA) were digested to the nucleoside level using the Nucleoside Digestion Mix (ref. M0649S) from New England BioLabs. To this reason, a solution of $70 \mathrm{ng}$ gDNA in $38 \mu \mathrm{L}$ of milliQ-water was prepared. As heavy-atom-labeled internal standards, $1.28 \mathrm{pmol} \mathrm{D}_{3}-\mathrm{mC}$ and $0.193 \mathrm{pmol} \mathrm{D}_{2}{ }^{15} \mathrm{~N}_{2}$-hmC in $6 \mu \mathrm{L}$ of milliQ-water were added to the solution, followed by $5 \mu \mathrm{L}$ of the Nucleoside Digestion Mix Reaction Buffer (10x), and $1 \mu \mathrm{L}$ of the Nucleoside Digestion Mix. After incubation for $90 \mathrm{~min}$ at $37^{\circ} \mathrm{C}$, the mixture was filtered using an AcroPrep Advance 96 filter plate $0.2 \mu \mathrm{m}$ Supor from Pall Life Sciences and subsequently analyzed by LC-MS. For each biological sample, two independent measurements (technical replicates) were performed. Quantitative LC-ESI-MS/MS analysis of the enzymatically digested DNA samples was performed using an Agilent 1290 UHPLC system coupled to an Agilent 6490 triple quadrupole mass spectrometer. The UHPLC-conditions used and the settings of the mass spectrometer were the same as previously published by Traube and colleagues. ${ }^{26}$

\section{Selective chemical labeling of $5 \mathrm{hmC}$ coupled with sequencing (5hmC-seq)}

For each sample, $550 \mathrm{ng}$ of genomic DNA was sonicated with a Bioruptor Pico in Tris $10 \mathrm{mM}$ pH 8 to obtain DNA fragments of $300 \mathrm{bp}$ in average. $25 \mathrm{pg}$ of $5 \mathrm{hmC}$ control spike-in were added to the sonicated DNA (control provided by the kit HydroxyMethylCollector, Active Motif, ref. 55013). $50 \mathrm{ng}$ of DNA was conserved at this stage to make the input library later. The remaining DNA was processed using the HydroxyMethylCollector kit (method from Song and colleagues 27 ) to glycosylate and biotinylate specifically the genomic $5 \mathrm{hmC}$. After glycosylation and biotinylation the DNA was purified with Ampure beads (Beckman Coulter, ref. A63881) . The DNA fragments containing the biot-glu-5hmC were purified with Streptavidine beads (Active Motif, ref. 55013), eluted, purified with Ampure beads and finally eluted in $50 \mathrm{uL}$ Tris $\mathrm{pH}$. The $5 \mathrm{hmC}$-seq libraries were prepared with the kit NEBNext Ultra II DNA library prep kit for Illumina (ref. E7645S) and indexed with NEBNext dual indexed primers (E7600S). The libraries were quality-checked by HS DNA Agilent BioAnalyzer (Supplementary Figure 1), dosed by DNA HS Qubit, pooled and submitted to the genome sequencing platform for Single-Read 50 bp Illumina HiSeq-2500 Rapid Run sequencing.

\section{RNA-seq librairies}

As the IFM-DFCl did not include RNA-seq data from patients at relapse, we produced RNA-seq data at diagnosis and at relapse on 4 selected MM patients (patients number MM_2, MM_5, MM_7 and MM_21). The RNA-seq libraries were prepared using the NEBNext Poly(A) mRNA Magnetic Isolation Module (NEB, ref. E74905) and the NEBNext Ultra II Directional RNA Library Prep Kit for Illumina (NEB, ref. E7760S) and sequenced by an Illumina Rapid Run HiSeq 2500 Single-Read 50 bp.

\section{Bioinformatics}

\section{5hmC-seq analysis}

$5 \mathrm{hmC}$-seq libraries were trimmed with Cutadapt v1.18 28 and mapped to human reference genome GRCh38 using Bowtie2 v2.2. $8^{29}$ with default parameters. Duplicates and reads of low mapping quality $(Q<30)$ were discarded. $5 \mathrm{hmC}$ peak clusters were defined similar to Boeva and colleagues 3031 with a stitching distance of $12.5 \mathrm{~kb}$ (Supplementary Figure 2). Blacklisted regions consist of chromosomes X, Y, ENCODE reference blacklist and peaks called on input-seq libraries.

Genes and $5 \mathrm{hmC}$ peaks clusters were associated to the Topologically Associated Domain (TAD) of the B-cell derived cell line GM12878 they are located in similar to Boeva and colleagues. ${ }^{31}$

Group specific $5 \mathrm{hmC}$ peaks clusters were identified by computing the average Log2 Fold-Change signal per region between groups and tested with a Wilcoxon rank-sum test adjusted with a Benjamini-Hochberg correction. For paired samples (two replicates of each diagnosis and relapse), we used DiffBind ${ }^{32}$ with default parameters to find differentially enriched $5 \mathrm{hmC}$ regions.

Potential transcription factor binding sites and core regulatory circuiteries were found with CRCMapper and MEME 3334 
Network clustering of $5 \mathrm{hmC}$ was computed on the correlation matrix of peak clusters in the cohort. A graph adjacency matrix was calculated from the correlation between peak clusters with a threshold of 0.715 . Every correlation above 0.715 is counted as an adjacency between two peak clusters. This value was chosen to limit the number of non-connected components in the graph and to keep about 400 peak clusters in the analysis. The two sub-networks were then identified from the hierarchical clustering (Ward's method) of the first two Eigen components of the symmetric normalized Laplacian matrix. ${ }^{35}$

Bioinformatics code is hosted at: https://gitlab.univ-nantes.fr/alberge/5hmc-myeloma-analysis.

\section{RNA-seq analysis}

IFM-DFCI RNA-seq data were obtained from Cleynen and colleagues ${ }^{36}$ or sequenced by us. RNA-seq libraries were trimmed using Cutadapt v1.13 $3^{28}$ with parameter $-m 20$ and aligned to hg38/GRCh38 using STAR v2.5.3a $a^{37}$ with default parameters. Genes were quantified using featureCounts ${ }^{38}$ from the R package Rsubread and the Gencode v28 genome annotation. RefSeq genome annotation was used in parallel as a reference for transcription factor selection.

\section{ChIP-seq and ATAC-seq analysis}

ChIP-seq and ATAC-seq libraries were downloaded from the European Nucleotide Archive (project number PRJEB2560539) and treated with the pyflow-ChIPseq 40 and pyflow-ATACseq pipelines respectively with default parameters. Chromatine-states genome annotation was realized using ChromHMM ${ }^{41}$ on ChIP-seq data from MM.1S cell line.

\section{Survival analysis}

Time-to-event was calculated from the randomization to the event date, i.e. death for OS, death or relapse for PFS, or to the last follow-up date.

For MS quantitative variables, cohorts were split at the median value of high and low $5 \mathrm{hmc}$ (and $5 \mathrm{mC}$ ). Survival curves were calculated using the Kaplan-Meier method and groups were compared using a Log-rank test.

For $5 \mathrm{hmC}$-seq data, at univariate step, $\mathrm{SAM}^{42}$ was run to extract the $5 \mathrm{hmC}$ regions that are significantly correlated with overall survival $\left(q_{\text {value }}<0.05\right)$ with 100 permutations and $\Delta=0.2$. For multiple regions testing (scores $\mathrm{SC1}$ and SC2), the cohort was split at median value of $5 \mathrm{hmC}$ enrichment. $5 \mathrm{hmC}$ of each patient was summarized by the weighted mean of independently significant parameters. Variables were standardized and weights were based on multivariate Cox's coefficients changed to +1 or -1 according to their sign to avoid overfitting. Survival curves were calculated using the Kaplan-Meier method and groups were compared using a Log-rank test.

\section{Data Sharing Statement}

Sequencing data are accessible at ENA under accession PRJEB32800. Mass spectrometry data are available in Supplemental Table.

\section{Results}

\section{The $5 \mathrm{hmC}$ regions landscape in $\mathrm{MM}$}

We studied a cohort of 40 patients newly diagnosed with MM between 2010-2012 and 5 healthy bone marrow donors (Figure 1A).

We quantified by MS the global level of $5 \mathrm{mC}$ and $5 \mathrm{hmC}$ in our 49 samples (40 diagnosis in $5 \mathrm{mC}, 39$ in $5 \mathrm{hmC}$, 4 relapses and 5 normal plasma cell samples). We found the $5 \mathrm{mC}$ and the $5 \mathrm{hmC}$ to be both significantly reduced in MM compared to normal plasma cells (NPC; Figure 1B and Supplementary Figure 3A). The amount of $5 \mathrm{hmC}$ is reduced in average by about 4 fold between NPC and MM ( $\left.\mathrm{p}_{\text {value }}<0.001\right)$. We found that $5 \mathrm{hmC}$, and not $5 \mathrm{mC}$, is reduced in MM Stage II/III compared to Stage I regarding to the International Staging System, ${ }^{43}$ a classification of patients based on $\beta_{2}$-microglobulin and albumin levels with a strong prognosis value ( $\mathrm{p}_{\text {value }}=0.025$, Figure $1 \mathrm{C}$ and Supplementary Figure $3 \mathrm{~B}$ ). $5 \mathrm{mC}$ and $5 \mathrm{hmC}$ global levels were not correlated to the sex or the age of the patients (Supplementary Figure 3C-3F).

These results encouraged us to characterize the hydroxymethylome by $5 \mathrm{hmC}-\mathrm{seq}^{27}$ to identify the residual genomic regions marked by $5 \mathrm{hmC}$ in $\mathrm{MM}$.

We confirm that the level of $5 \mathrm{hmC}$ signal in enriched at gene bodies and is positively associated with RNA expression as expected from previous studies20 $2744-46$ (Figure 1D).

The $5 \mathrm{hmC}$ signal is particularly enriched at strong enhancers and to a lesser extend at weak enhancers (Figure $1 \mathrm{E})$ when we used a functional annotation from the MM.1S cell line. 
Inspection of regions of interest showed that strong levels of $5 \mathrm{hmC}$ can be associated between distal loci (Figure $1 \mathrm{~F})$. For instance at the CCND2 locus, we found a strong correlation $\left(R^{2}=0.88\right)$ between $5 \mathrm{hmC}$ signal in the gene body (chr12:4,278,700-4,312,900) and 5hmC signal at an extragenic centromeric region located $120 \mathrm{~kb}$ upstream (chr12:4,106,500-4,164,700; Supplementary Figure 4A). Both 5hmC signals correlate well with CCND2 RNA expression and both regions are located in the same topogical domain according to $\mathrm{HiC}$ data 47 48 (chr12:3,850,0004,800,000; Supplementary Figure 4B). The upstream region is also marked by ATAC and H3K27ac signals in MM patients ${ }^{39}$ suggesting that this genomic region is functionally active. This strongly suggests that this upstream region is an enhancer of CCND2 gene.

Hence we pursued the analyses using $18165 \mathrm{hmC}$ peak clusters. We determined the $5 \mathrm{hmC}$ peak clusters common to at least two patients and performed an unsupervised Principal Component Analysis (PCA). PCA plot shows a great heterogeneity of MM compared with Normal patients (Figure 2A). PC1 is driven by CCND1 (x-axis, left-side) versus CCND2 (x-axis, right-side). PC2 is driven by CCND1 (y-axis, bottom) versus FGFR3-MMSET (y-axis, top) (Supplementary Table), the three loci being of major interest in MM. Patients samples were then classified in 5 groups: MMSET (translocation $\mathrm{t}(4 ; 14)$; 9 patients), CCND1 (RNA expression over 800 Transcripts per Million; 11 patients), hyperdiploid (16 patients, at least 2 odd chromosomal gains), others (MM in none of the aforementioned groups; 4 patients) and normal plasma cells (Normal; 5 donors).

For each group, we determined specific $5 \mathrm{hmC}$ peak clusters (Figures 2B, 2C, Supplementary Figure 5A and Supplementary Table). Remarkably, the strongest specific $5 \mathrm{hmC}$ peak cluster for the group MMSET is the locus FGFR3-MMSET ( $\mathrm{p}_{\text {value }}=1.610^{-6}$ ) followed by CCND2, LILRB4, NBEA and TRMT9B (Figure 2D and Supplementary Figure $5 \mathrm{~B}$ ). This strong $5 \mathrm{hmC}$ enrichment in MMSET patients is also associated with strong H3K27ac and ATAC-seq signals ${ }^{39}$ compared to the other MM patients. This result suggests that the translocation between the FGFR3MMSET locus and the $\mathrm{IgH}$ locus is associated massive $5 \mathrm{mC}$ oxidation together with chromatin opening and very high transcription level (Figure 2D).

Remarkably, in patients from the CCND1 group, the strongest and most specific $5 \mathrm{hmC}$ peak cluster was located in the CCND1 gene itself (Figure $2 \mathrm{C}$ and Supplementary Figures $5 \mathrm{C}, \mathrm{p}_{\text {value }}=0.05$ ). Again this suggests that the translocation event between $C C N D 1$ and $\operatorname{lgH}$ or the novel overexpression of CCND1 induces very high $5 \mathrm{mC}$ oxidation together with a strong CCND1 transcription activity even though the classification of CCND1 patients was done based on RNA level in absence of FISH data.

The hyperdiploid group shows strongly specific $5 \mathrm{hmC}$ signal at $H G F\left(\right.$ Hepatocyte growth factor, $\left.\mathrm{p}_{\text {value }}=0.02\right)$ and at the locus of $M Y C$ oncogene (Figure 2B, 2C, Supplementary Figure 5D, $p_{\text {value }}=0.02$ ).

Normal plasma cells are enriched in 5hmC at BTNL8, C11orf80, ITM2C, PSG4 and TRIO genes (Figure 2B, 2C).

We found high $5 \mathrm{hmC}$ signal together with $\mathrm{H} 3 \mathrm{~K} 27 \mathrm{ac}$ and ATAC signals ${ }^{39}$ at several important genes implicated in MM including CCND1, FGFR3-MMSET, IRF4, PRDM1 and XBP1 (Supplementary Figures 6A-F). Specifically, H3K27ac super-enhancers from a patient with $\mathrm{t}(4 ; 14)$, del13 and amp1q from Jin and colleagues ${ }^{39}$ and the $5 \mathrm{hmC}$ peak clusters found in one of our patients with the same genomic alterations are located at similar genomic loci CREB3L2, FNDC3B, MMSET, ST6GAL1 and ZBTB38 (Supplementary Figures 5E,F).

\section{Transcriptional regulatory networks in MM}

To uncover transcriptional regulation networks shaping multiple myeloma, we next measured the signal correlation between the $18165 \mathrm{hmC}$ peak clusters among the 40 patients and searched for correlation networks. A correlation matrix of $4075 \mathrm{hmC}$ peak clusters was obtained, in which 2 correlation networks (network 1 and network 2, Figure $3 \mathrm{~A}$ ) were defined through hierarchical clustering (of resp. 242 and $1655 \mathrm{hmC}$ peak clusters). To find the core transcription factors (TF) involved in these 2 networks, we did a core transcriptional regulatory circuits analysis ${ }^{34}$ for each patient based on $5 \mathrm{hmC}$ and RNA expression data. We found that core TFs motifs were differently enriched between the 2 networks. Network 1 was enriched with TFs motifs such as KLF6/13, MYC, SP3, SREBF2, TCF3, USF2 and ZBTB7A, while network 2 was enriched with ARID3A, ATF4, FOXO3, IRF4/7, NFIL3 and PRDM1 motifs (Figures 3B, 3C, 3E). The ten most expressed TF were ATF4, KLF6/13, IRF1/4, PRDM1, TCF3, USF1/2 and XBP1 (Supplementary Figure 7A). These core transcription factors are shared between the two networks (Supplementary Figure 7B). For each patient, we averaged $5 \mathrm{hmC}$ signal in networks 1 and 2 and found the patients to be continuously distributed in terms of network scores rather than being clustered in one of the networks (Figure 3D). Through GREAT analysis, ${ }^{49}$ network 1 was shown to be associated with negative regulation of cytokine production and negative regulation of tumor necrosis factor production, while the network 2 was associated with immune response-regulating cell surface receptor signaling pathway and immune response-regulating signaling pathway (Figure 3F). Network analysis suggests that MM could be shaped by 2 regulation networks associated with specific transcription factors with partially redundant functions.

\section{5hmC dynamics between diagnosis and relapse in MM}

To identify the genomic regions associated with MM progression, we next mapped 5hmC in 4 MM pairs (diagnosis and first relapse) and identified differentially hydroxymethylated regions with two replicates for each condition (Figure 
4A). CNV microarray data ${ }^{50}$ a1 and RNA-seq were used at both time points to assess the progression of each patient (Figure 4B).

The plasma cells of patient MM_2 showed a translocation of the MMSET locus $(\mathrm{t}(4 ; 14))$ with a deletion of chromosomes $1 \mathrm{p}, 13$ and $17 \mathrm{p}$ at diagnosis. This subject progressed in 18 months, with his plasma cells displaying a third copy of $1 \mathrm{q}$. Out of 560 consensus $5 \mathrm{hmC}$ regions, $269(48 \%)$ are significantly reduced at relapse compared to diagnosis $(\mathrm{FDR}<0.05)$ while $20(3.5 \%)$ are enriched at relapse, suggesting a global loss of $5 \mathrm{hmC}$. Interestingly, we noted a significant gain of $5 \mathrm{hmC}$ at CCND2 and IKZF1 genes bodies at relapse (respectively 1,6 and 1,5 fold) associated with a higher transcriptional activity (respectively 2,4 and 1,5 fold). The drug lenalidomide induces IKZF1 proteasomal degradation, 5253 suggesting that the up-regulation of $I K Z F 1$, together with an increase in CCND2 expression could favor disease progression. We also noticed a gain of $5 \mathrm{hmC}$ at MAPKAPK2/MK2 at relapse. This gene is located on 1q, which is gained at relapse by this patient, and has been recently described as a poor prognosis factor 54

At diagnosis, patient MM_5 displayed a classical hyperdiploid profile with amplification of chromosomes 3, 9, 11, 15,19 , where 675 regions were found to harbor $5 \mathrm{hmC}$ peak clusters. At relapse 24 months later, cells displayed a 1-copy loss of $17 \mathrm{p}$, with 22 regions (3.2\%) significantly decreased. Accordingly to TP53 loss, expression and $5 \mathrm{hmC}$ signal of p53 target genes such as MDM2 was found decreased (Figure 4C)!55 56

Patient MM_7 is another MMSET translocated patient $(\mathrm{t}(4 ; 14))$ with amplification of chromosome arm $1 \mathrm{q}$ and deletion of chromosomes 13 and $17 p$ (Figure 4B) gaining deletions at relapse (3p, 6q, 8q). At relapse, 5hmC increases at several genes such as CDKN2C, IGF2BP3 and WNT5B as well as their RNA expression (log fold-change $>3$ ).

Patient MM_21 plasma cells were found to have a narrow TET2 deletion at diagnosis, but surprisingly 2 heterozygous copies at relapse. TET2, which converts $5 \mathrm{mC}$ to $5 \mathrm{hmC}$, is frequently mutated in cancer and especially in myeloid malignancies,, 57 but not in $\mathrm{MM} \stackrel{4}{485}$ regions were found to harbour $5 \mathrm{hmC}$, out of which $86(22 \%)$ decreased and $117(30 \%)$ increased at relapse. Global level of $5 \mathrm{hmC}$ decreased moderately despite the reappearance of TET2 (Supplementary Table).

\section{$5 \mathrm{hmC}$ and survival in $\mathrm{MM}$}

Survival analysis was first performed on the global level of $5 \mathrm{mC}$ and $5 \mathrm{hmC}$. $5 \mathrm{hmC}$ (indicated as $5 \mathrm{hmC} / \mathrm{dG}[\%]$ ) tends to be associated with Overall Survival (OS) although statistically non-significant (Hazard ratio (HR) $=2.6$, $\left.\mathrm{Cl}=[0.9,7.8], \mathrm{p}_{\text {value }}=0.066\right)$ (Figure $5 \mathrm{~A}$ ). Yet $5 \mathrm{hmC}$ is less likely to be associated with Progression-Free Survival (PFS; Figure $5 B$; $p_{\text {value }}=0.3$ ). $5 \mathrm{mC}$ global level does not show significant association with OS and PFS (Supplementary Figures $8 \mathrm{~A}$ and $8 \mathrm{~B})$.

Three $5 \mathrm{hmC}$ genomic loci were found to separate independently the patients in 2 groups of good and poor survival. High $5 \mathrm{hmC}$ signal at the TP53INP1 gene is associated with longer survival $\left(\mathrm{HR}=3.7, \mathrm{Cl}=[1.2-11], \mathrm{p}_{\text {value }}=0.017\right.$; Supplementary Figure $8 \mathrm{C}$ ) and low $5 \mathrm{hmC}$ signal at RNF144A gene (or UPF1 gene) is associated with poorer survival (data not shown).

The 3 independent $5 \mathrm{hmC}$ regions (RNF144A, TP53INP1 and UPF1) were then summarized into a score SC1 (see Methods) that separates the cohort into 2 median groups of 20 patients of bad and good outcomes (HR=12, $\mathrm{Cl}=[4,244], \mathrm{p}_{\text {value }}=110^{-6}$ for $\mathrm{OS} ; \mathrm{HR}=3.8, \mathrm{Cl}=[1.7,8.6], \mathrm{p}_{\text {value }}=410^{-4}$ for PFS; Figures $5 \mathrm{C}$ and 5D). The lower risk group shows higher level of $5 \mathrm{hmC}$ at TP53INP1 gene and lower levels at RNF144A and UPF1 genes. When applied to gene expression of RNF144A TP53INP1 and UPF1, no significant impact on survival was found (HR=1.4, $\mathrm{Cl}=[0.27,2.0]$, p value $=0.5$; Supplementary Figure 8D).

We found that $5 \mathrm{hmC}$ global level is independent of the $5 \mathrm{hmC}$ enrichment at the 3 genomic loci. We combined the 4 variables into a second score SC2 (see Methods). Patients with better outcome show high global level of $5 \mathrm{hmC}$, high $5 \mathrm{hmC}$ signal at TP53INP1 and low $5 \mathrm{hmC}$ signals at $R N F 144 A$ and UPF1 $\left(\mathrm{HR}=24, \mathrm{Cl}=[3.2,187], \mathrm{p}_{\text {value }}=7\right.$ $10^{-6}$ for $\mathrm{OS} ; \mathrm{HR}=3.8, \mathrm{Cl}=[1.7,8.4], \mathrm{p}_{\text {value }}=610^{-4}$ for PFS; Figures 5E and 5F).

\section{Discussion}

We have quantified the epigenetic marks $5 \mathrm{mC}$ and $5 \mathrm{hmC}$ and mapped $5 \mathrm{hmC}$ in plasma cell DNA samples from 40 newly diagnosed MM patients and 5 donors. The global levels of $5 \mathrm{mC}$ and $5 \mathrm{hmC}$ are lower in MM compared to NPC. $5 \mathrm{hmC}$ is higher in Stage I MM compared to Stage II and Stage III patients. 5hmC tends to be positively associated with survival. These observations show that $5 \mathrm{hmC}$ is a marker of disease progression and severity.

Despite a global hypohydroxymethylation in $\mathrm{MM}$, there still remain $5 \mathrm{hmC}$ marks at transcriptionally active regions. We revealed that $5 \mathrm{hmC}$ is strongly associated with active enhancers and identified active genomic regions specific to known MM molecular groups. We also found that H3K27ac-based super-enhancers from Jin and colleagues ${ }^{39}$ share similarities with our $5 \mathrm{hmC}$ peak clusters. These observations confirm that $5 \mathrm{hmC}$ is a valuable mark to study active genomic regions.

An unsupervised analysis shows that $5 \mathrm{hmC}$ at major oncogenes (CCND1, CCND2, FGFR3 and MMSET) can separate hydroxymethylomes of MM patients, consistent with the existing classifications based on genetic abnormalities. 
5hmC signals at the loci CCND1 and FGFR3-MMSET are strong markers of the CCND1 and MMSET molecular groups respectively. This suggests that the translocation events are the major factors shaping MM hydroxymethylome.

We discovered 2 networks of highly correlated $5 \mathrm{hmC}$ regions that offer a new prism to view MM transcriptional regulation. Our analysis connects target genes and regulatory regions to transcription factors. The 2 networks are predicted to be controlled by key transcription factors of well-known importance in MM (IRF4, MYC, PRDM1, and XBP1) as well as novel genes associated with the disease regulation (ATF4, IRF7, and TCF3). Of note, several MM-related transcription factors are under investigation to be drug-targeted and represent promising therapeutic targets. ${ }^{58}$ The gradual distribution of $5 \mathrm{hmC}$ signal between the two networks suggests that they may have redundant functions.

Between diagnosis and relapse, we found a highly dynamic and patient-specific distribution of the $5 \mathrm{hmC}$ signal. This reflects that MM progression is highly heterogeneous, although we could find consistency between $5 \mathrm{hmC}$ changes, expression and copy number variations.

We found $35 \mathrm{hmC}$ regions that were independently associated with overall survival. A high $5 \mathrm{hmC}$ signal at the TP53INP1 gene is associated with a longer survival while low $5 \mathrm{hmC}$ signals at RNF144A and UPF1 genes are associated with good outcome. TP53INP1 protein is shown as a tumor suppressor with antiproliferative and proapoptotic functions. 5960 Interestingly, the association of TP53INP1 and overall survival is independent of del17p status.

Our study shows that the epigenetic mark $5 \mathrm{hmC}$ is valuable to discover active regulatory regions in genomic DNA from a cohort of patients without the need of chromatin extraction. It has been recently shown that it is also possible to map $5 \mathrm{hmC}$ on circulating DNA. ${ }^{22} 23$ This makes $5 \mathrm{hmC}$ not only biologically valuable but also technically easier than ChIP-seq against histone marks or transcription factors. Taken together, these results show the value of epigenomics in retrospective studies and bring to light potential drug targets that drive the malignant transcriptome.

\section{Acknowledgments}

We thank G. Salbert for the critical reading of this manuscript. We thank M. Devic, E. Douillard, E. Ollivier and N. Roi for their great technical support. We thank the Biogenouest sequencing platform GenoBird from Nantes for the Illumina sequencing. We thank the medical staff of the Nouvelles Cliniques Nantaises - Le Confluent that provided the bone marrow cells collected during hip replacement surgery.

This study was supported by the Chaire Mixte INSERM - Ecole Centrale de Nantes, the Intergroupe Francophone du Myelome, the Ligue Contre le Cancer, the I-SITE NexT (ANR-16-IDEX-0007) and the SIRIC ILIAD (INCa-DGOSInserm-12558). AJ was supported by a PhD Fellowship from INSERM and Région Pays de Loire. Research of M.W. and T.C. was supported by the Deutsche Forschungsgemeinschaft (SFB1309: TP A04, SFB1361: TP 02) and the European Research Council (ERC-AG) under the European Union's Horizon 2020 research and innovation program (Project ID: EpiR: 741912).

\section{Authorship Contributions}

J.-B.A., F.M., S.M, and A.S designed the project and analyzed the data. M.A., H.A.-L., P.M and S.M. created the cohort and provided patient samples. A.S. and S.D. produced the $5 \mathrm{hmC}$-seq data. M.W. and T.C. performed the quantitative mass spectrometry measurements and interpreted the data. J.-B.A., C.G. and L.C. analyzed the survival data. A.S. and J.-B.A. wrote the manuscript. All authors read and corrected the manuscript.

\section{Disclosure of Conflicts of Interest}

The authors declare no competing interests.

\section{References}

${ }^{1}$ Kumar S. K., Dispenzieri A., Lacy M. Q., et al. Continued improvement in survival in multiple myeloma: changes in early mortality and outcomes in older patients. Leukemia. 2014;28:1122-1128.

${ }^{2}$ Moreau P., Attal M., Hulin C., et al. Bortezomib, thalidomide, and dexamethasone with or without daratumumab before and after autologous stem-cell transplantation for newly diagnosed multiple myeloma (CASSIOPEIA): a randomised, open-label, phase 3 study. Lancet. 2019;394:29-38.

${ }^{3}$ Manier S., Salem K. Z., Park J., Landau D. A., Getz G., Ghobrial I. M.. Genomic complexity of multiple myeloma and its clinical implications. Nat Rev Clin Oncol. 2017;14:100-113. 
${ }^{4}$ Morgan G. J., Walker B. A., Davies F. E.. The genetic architecture of multiple myeloma. Nat Rev Cancer. 2012;12:335-348.

${ }^{5}$ Nutt S. L., Hodgkin P. D., Tarlinton D. M., Corcoran L. M.. The generation of antibody-secreting plasma cells. Nat Rev Immunol. 2015;15:160-171.

${ }^{6}$ Agirre X., Castellano G., Pascual M., et al. Whole-epigenome analysis in multiple myeloma reveals DNA hypermethylation of B cell-specific enhancers. Genome Res. 2015;25:478-487.

${ }^{7}$ Chatonnet F., Pignarre A., Sérandour A. A., et al. The hydroxymethylome of multiple myeloma identifies FAM72d as a 1q21 marker linked to proliferation. Haematologica. 2019:haematol.2019.222133.

8 Tahiliani M., Koh K. P., Shen Y., et al. Conversion of 5-methylcytosine to 5-hydroxymethylcytosine in mammalian DNA by MLL partner TET1. Science. 2009;324:930-935.

${ }^{9}$ Kriaucionis S., Heintz N.. The nuclear DNA base 5-hydroxymethylcytosine is present in purkinje neurons and the brain. Science. 2009;324:929-930.

${ }^{10}$ Ito S., D'Alessio A. C., Taranova O. V., Hong K., Sowers L. C., Zhang Y.. Role of Tet proteins in 5mc to 5hmc conversion, ES-cell self-renewal and inner cell mass specification. Nature. 2010;466:1129-1133.

${ }^{11}$ He Y.-F., Li B.-Z., Li Z., et al. Tet-mediated formation of 5-carboxylcytosine and its excision by TDG in mammalian DNA. Science. 2011;333:1303-1307.

12 Shen L., Wu H., Diep D., et al. Genome-wide analysis reveals TET- and TDG-dependent 5-methylcytosine oxidation dynamics. Cell. 2013;153:692-706.

${ }^{13}$ Globisch D., Münzel M., Müller M., et al. Tissue distribution of 5-hydroxymethylcytosine and search for active demethylation intermediates. PLoS One. 2010;5:e15367.

${ }^{14}$ Haffner M. C., Chaux A., Meeker A. K., et al. Global 5-hydroxymethylcytosine content is significantly reduced in tissue stem/progenitor cell compartments and in human cancers. Oncotarget. 2011;2.

${ }^{15}$ Nestor C. E., Ottaviano R., Reddington J., et al. Tissue type is a major modifier of the 5-hydroxymethylcytosine content of human genes. Genome Res. 2012;22:467-477.

16 Kohli R. M., Zhang Y.. TET enzymes, TDG and the dynamics of DNA demethylation. Nature. 2013;502:472-479.

${ }^{17}$ Bachman M., Uribe-Lewis S., Yang X., Williams M., Murrell A., Balasubramanian S.. 5-Hydroxymethylcytosine is a predominantly stable DNA modification. Nat Chem. 2014;6:1049-1055.

${ }^{18}$ Bachman M., Uribe-Lewis S., Yang X., et al. 5-Formylcytosine can be a stable DNA modification in mammals. Nat Chem Biol. 2015;11:555-557.

${ }^{19}$ Stroud H., Feng S., Morey Kinney S., Pradhan S., Jacobsen S. E.. 5-Hydroxymethylcytosine is associated with enhancers and gene bodies in human embryonic stem cells. Genome Biol. 2011;12:R54.

${ }^{20}$ Sérandour A. A., Avner S., Oger F., et al. Dynamic hydroxymethylation of deoxyribonucleic acid marks differentiation-associated enhancers. Nucleic Acids Res. 2012;40:8255-8265.

${ }^{21}$ Mahé E. A., Madigou T., Sérandour A. A., et al. Cytosine modifications modulate the chromatin architecture of transcriptional enhancers. Genome Res. 2017;27:947-958.

${ }^{22} \mathrm{Li}$ W., Zhang X., Lu X., et al. 5-Hydroxymethylcytosine signatures in circulating cell-free DNA as diagnostic biomarkers for human cancers. Cell Res. 2017;27:1243-1257.

${ }^{23}$ Song C.-X., Yin S., Ma L., et al. 5-Hydroxymethylcytosine signatures in cell-free DNA provide information about tumor types and stages. Cell Res. 2017;27:1231-1242.

24 The International Myeloma Working Group*. Criteria for the classification of monoclonal gammopathies, multiple myeloma and related disorders: a report of the International Myeloma Working Group. Br J Haematol. 2003;121:749-757.

${ }^{25}$ Attal M., Lauwers-Cances V., Hulin C., et al. Lenalidomide, bortezomib, and dexamethasone with transplantation for myeloma. N Engl J Med. 2017;376:1311-1320.

26 Traube F. R., Schiffers S., Iwan K., et al. Isotope-dilution mass spectrometry for exact quantification of noncanonical DNA nucleosides. Nat Protoc. 2019;14:283-312. 
${ }^{27}$ Song C.-X., Szulwach K. E., Fu Y., et al. Selective chemical labeling reveals the genome-wide distribution of 5-hydroxymethylcytosine. Nat Biotechnol. 2011;29:68-72.

${ }^{28}$ Martin M.. Cutadapt removes adapter sequences from high-throughput sequencing reads. EMBnet.journal. $2011 ; 17: 10$.

${ }^{29}$ Langmead B., Salzberg S. L.. Fast gapped-read alignment with Bowtie 2. Nat Methods. 2012;9:357-359.

${ }^{30}$ Ashoor H., Hérault A., Kamoun A., et al. HMCan: a method for detecting chromatin modifications in cancer samples using ChIP-seq data. Bioinformatics. 2013;29:2979-2986.

${ }^{31}$ Boeva V., Louis-Brennetot C., Peltier A., et al. Heterogeneity of neuroblastoma cell identity defined by transcriptional circuitries. Nat Genet. 2017;49:1408-1413.

32 Ross-Innes C. S., Stark R., Teschendorff A. E., et al. Differential oestrogen receptor binding is associated with clinical outcome in breast cancer. Nature. 2012;481:389-393.

33 Bailey T. L., Boden M., Buske F. A., et al. MEME Suite: tools for motif discovery and searching. Nucleic Acids Res. 2009;37:W202-W208.

${ }^{34}$ Saint-André V., Federation A. J., Lin C. Y., et al. Models of human core transcriptional regulatory circuitries. Genome Res. 2016;26:385-396.

${ }^{35}$ Hastie T., Tibshirani R., Friedman J.. The Elements of Statistical Learning. Springer Series in StatisticsNew York, NY: Springer New York 2009.

${ }^{36}$ Cleynen A., Szalat R., Samur Kemal M., et al. Expressed fusion gene landscape and its impact in multiple myeloma. Nat Commun. 2017;8:1-10.

37 Dobin A., Davis C. A., Schlesinger F., et al. STAR: ultrafast universal RNA-seq aligner. Bioinformatics. 2013;29:1521.

${ }^{38}$ Liao Y., Smyth G. K., Shi W.. featureCounts: an efficient general purpose program for assigning sequence reads to genomic features. Bioinformatics. 2014;30:923-930.

39 Jin Y., Chen K., De Paepe A., et al. Active enhancer and chromatin accessibility landscapes chart the regulatory network of primary multiple myeloma. Blood. 2018;131:2138-2150.

40 Terranova C., Tang M., Orouji E., et al. An integrated platform for genome-wide mapping of chromatin states Using High-throughput ChIP-sequencing in Tumor Tissues. J Vis Exp. 2018.

${ }^{41}$ Ernst J., Kellis M.. ChromHMM: automating chromatin-state discovery and characterization. Nat Methods. 2012;9:215-216.

42 Tusher V. G., Tibshirani R., Chu G.. Significance analysis of microarrays applied to the ionizing radiation response. Proc Natl Acad Sci U S A. 2001;98:5116-5121.

${ }^{43}$ Greipp P. R., Miguel J. S., Durie B. G., et al. International staging system for multiple myeloma. J Clin Oncol. 2005;23:3412-3420.

${ }^{44}$ Ficz G., Branco M. R., Seisenberger S., et al. Dynamic regulation of 5-hydroxymethylcytosine in mouse ES cells and during differentiation. Nature. 2011;473:398-402.

${ }^{45}$ Williams K., Christensen J., Pedersen M. T., et al. TET1 and hydroxymethylcytosine in transcription and DNA methylation fidelity. Nature. 2011;473:343-348.

${ }^{46} \mathrm{Wu}$ H., D'Alessio A. C., Ito S., et al. Genome-wide analysis of 5-hydroxymethylcytosine distribution reveals its dual function in transcriptional regulation in mouse embryonic stem cells. Genes Dev.. 2011;25:679-684.

${ }^{47}$ Rao S. S. P., Huntley M. H., Durand N. C., et al. A 3d map of the human genome at kilobase resolution reveals principles of chromatin looping. Cell. 2014;159:1665-1680.

${ }^{48}$ Wang Y., Song F., Zhang B., et al. The 3d Genome Browser: a web-based browser for visualizing 3d genome organization and long-range chromatin interactions. Genome Biol. 2018;19:151.

${ }^{49}$ McLean C. Y., Bristor D., Hiller M., et al. GREAT improves functional interpretation of cis-regulatory regions. Nat Biotechnol. 2010;28:495-501. 
${ }^{50}$ Magrangeas F., Avet-Loiseau H., Gouraud W., et al. Minor clone provides a reservoir for relapse in multiple myeloma. Leukemia. 2013;27:473-481.

${ }^{51}$ Magrangeas F., Kuiper R., Avet-Loiseau H., et al. A genome-wide association study identifies a novel locus for bortezomib-induced peripheral neuropathy in european patients with multiple myeloma. Clin Cancer Res. 2016;22:4350-4355.

52 Krönke J., Udeshi N. D., Narla A., et al. Lenalidomide causes selective degradation of IKZF1 and IKZF3 in multiple myeloma cells. Science. 2014;343:301-305.

${ }^{53}$ Lu G., Middleton R. E., Sun H., et al. The myeloma drug lenalidomide promotes the cereblon-dependent destruction of Ikaros proteins. Science. 2014;343:305-309.

${ }^{54} \mathrm{Gu}$ C., Cheng H., Yang H., et al. MK2 is a therapeutic target for high-risk multiple myeloma. Haematologica. 2018:haematol.2017.182121.

${ }^{55}$ Haupt Y., Maya R., Kazaz A., Oren M.. Mdm2 promotes the rapid degradation of p53. Nature. 1997;387:296-299.

${ }^{56}$ Kubbutat M. H. G., Jones S. N., Vousden K. H.. Regulation of p53 stability by Mdm2. Nature. 1997;387:299-303.

${ }^{57}$ Ko M., Huang Y., Jankowska A. M., et al. Impaired hydroxylation of 5-methylcytosine in myeloid cancers with mutant TET2. Nature. 2010;468:839-843.

58 Li S., Vallet S., Sacco A., Roccaro A., Lentzsch S., Podar K.. Targeting transcription factors in multiple myeloma: evolving therapeutic strategies. Expert Opin Investig Drugs. 2019;28:445-462.

${ }^{59}$ Seillier M., Peuget S., Gayet O., et al. TP53inp1, a tumor suppressor, interacts with LC3 and ATG8-family proteins through the LC3-interacting region (LIR) and promotes autophagy-dependent cell death. Cell Death Differ. 2012;19:1525-1535.

60 Sancho A., Duran J., García-España A., et al. DOR/Tp53inp2 and Tp53inp1 Constitute a Metazoan Gene Family Encoding Dual Regulators of Autophagy and Transcription. PLoS One. 2012; 7:e34034.

\section{Figure Legends}

Figure 1: $5 \mathrm{hmC}$ is associated with patient classification and is located at expressed genes and at strong enhancers. (A) Experimental pipeline leading to $\mathrm{MS}$ and $5 \mathrm{hmC}$-seq analysis in plasma cells from patient bone marrows. (B) Dot plot of $5 \mathrm{hmC}$ global quantification by $\mathrm{MS}$ in normal plasma cells from healthy donors $(\mathrm{N}=5)$, and of myeloma cells of patients at diagnosis $(\mathrm{N}=39)$. (C) Dot plot of $5 \mathrm{hmC}$ global quantification by $\mathrm{MS}$ by disease stage (ISS I $\mathrm{N}=9$; ISS II $\mathrm{N}=17$; ISS III $\mathrm{N}=12 ; \mathrm{NA}=1$ ). ISS, International Staging System. (D) Average 5hmC signal at genes across the whole $5 \mathrm{hmC}$ dataset. The average $5 \mathrm{hmC}$ signal is plotted by class of gene expression level in RPKM. (E) Distribution of the $5 \mathrm{hmC}$-enriched regions at the different ChromHMM chromatin states across the $5 \mathrm{hmC}$ dataset. (F) 5hmC, ATAC, H3K27ac signal and ChromHMM states at the CCND2 genomic locus. The $5 \mathrm{hmC}$ signal correlation between the CCND2 gene and its putative enhancer is indicated. ATAC and H3K27ac data were published by Jin and colleagues. 39

Figure 2: $5 \mathrm{hmC}$ peak cluster are located at major functional genes in $\mathrm{MM}$ and can be associated with specific clinical subgroups. (A) $5 \mathrm{hmC}$ signal PCA plot showing the $40 \mathrm{MM}$ samples (groups MMSET, CCND1, Hyperdiploid and Other) and the 5 normal plasma cells (group Normal). (B) Heatmap showing the $5 \mathrm{hmC}$ signal at the most differential $5 \mathrm{hmC}$ peak clusters between the groups MMSET, CCND1, Hyperdiploid and Normal. Asterisks stand for proximal non-genic loci. (C) Plot showing the $5 \mathrm{hmC}$ peak clusters that are specifically enriched in patient groups MMSET, CCND1, Hyperdiploid and Normal. Significant regions are colored. (D) 5hmC, ATAC and H3K27ac signals at the FGFR3-MMSET locus in the MMSET patient group and the other patients.

Figure 3: $5 \mathrm{hmC}$ peak clusters are divided in two anti-correlated regulation networks. (A) Heatmap representing the signal correlation between the $5 \mathrm{hmC}$ peak clusters (407 correlated out of 1816). Clustering isolates two sets of regions that we called network 1 and network 2. (B) Motif enrichment of the core transcription factors in network 1 and 2 identified from the core transcriptional regulatory circuits analysis. (C) Network representation of the $5 \mathrm{hmC}$ peak clusters linked with the strongest correlations. Each dot is a $5 \mathrm{hmC}$ peak cluster. Edges are represented if the correlation between 2 nodes is greater than 0.715 . Color intensity represents the normalized number of motifs TCF3-MYC in the $5 \mathrm{hmC}$ peak clusters. (D) Scoring of patient samples in both networks 1 and 2 with linear regression. (E) IRF4-PRDM1 motif occurences in the networks representation. (F) Gene ontologies predicted by GREAT49 for the networks 1 and 2 .

Figure 4: $5 \mathrm{hmC}$ is dynamic and heterogeneous in $\mathrm{MM}$ between diagnosis and relapse. (A) Differential signal analysis of $5 \mathrm{hmC}$ peak clusters between diagnosis and relapse in patients MM_2, MM_5, MM_7 and MM_21. (B) Copy number variation history of MM patients between diagnosis and relapse. (C) $5 \mathrm{hmC}$ and RNA expression 
levels of the MDM2 gene at diagnosis and relapse in patient MM_5. At relapse, this patient acquires the deletion of the chromosome arm 17p (including a single-copy deletion of TP53).

Figure 5: Genomic 5hmC levels are associated with Overall and Progression-Free Survival. Univariate and multivariate Overall Survival (OS) and Progression-Free Survival (PFS) analysis. 5hmC enrichment is split at the median value (for univariate) or median value of weighted mean (for multivariate, see Methods). (A) OS over global level of $5 \mathrm{hmC}$ in MS. (B) PFS over global level of $5 \mathrm{hmC}$ in MS. (C) OS over $5 \mathrm{hmC}$ enrichment at TP53INP1, UPF1 and RNF144A genomic loci. (D) PFS over 5hmC enrichment at TP53INP1, UPF1 and RNF144A genomic loci. (E) OS combining global level of $5 \mathrm{hmC}$ in $\mathrm{MS}$ and $5 \mathrm{hmC}$ enrichment at TP53INP1, UPF1 and RNF144A genomic loci. (F) PFS combining global level of $5 \mathrm{hmC}$ in $\mathrm{MS}$ and $5 \mathrm{hmC}$ enrichment at TP53INP1, UPF1 and RNF144A genomic loci. (Time: Number of years).

\section{Supplementary Figure Legends}

Supplementary Figure 1: Agilent BioAnalyzer profile of a $5 \mathrm{hmC}$ Illumina library.

Supplementary Figure 2: Criteria of $\mathbf{5 h m C}$ peaks to merge in $\mathbf{5 h m C}$ peak clusters. The y-axis represents the number of peaks left after merging. The $x$-axis represents the distance between peaks to merge. Each $5 \mathrm{hmC}$ samples were analyzed (one color per patient). The distance $12.5 \mathrm{~kb}$ was chosen to define the $5 \mathrm{hmC}$ peak clusters that we used in this study.

Supplementary Figure 3: MS quantification of $5 \mathrm{mC}$ in genomic plasma cell DNA is independent of age and sex. (A) Dot plot of $5 \mathrm{mC}$ global quantification by $M S$ in normal plasma cells from healthy donors $(N=5)$, and of myeloma cells of patients at diagnosis $(N=40)$. (B) Dot plot of $5 \mathrm{hmC}$ global quantification by $\mathrm{MS}$ by disease stage (ISS I $\mathrm{N}=9$; ISS II $\mathrm{N}=17$; ISS III $\mathrm{N}=13$; $\mathrm{NA}=1$ ). $5 \mathrm{mC}(\mathrm{C})$ and $5 \mathrm{hmC}$ (D) dot plot of MS quantification depending on the sex of the patients. $5 \mathrm{mC}(\mathrm{E})$ and $5 \mathrm{hmC}(\mathrm{F})$ dot plot of MS quantification depending on the age of the patients.

Supplementary Figure 4: $5 \mathrm{hmC}$ allows the identification of a putative CCND2 enhancer. (A) Correlation between CCND2 expression, $5 \mathrm{hmC}$ at CCND2 gene body and $5 \mathrm{hmC}$ at the putative $5 \mathrm{hmC}$ enhancer across the 40 MM patients. (B) Hi-C signal in lymphoblastoid cells (GM12878 cells) at the CCDN2 locus showing the spatial interaction between CCND2 gene and its putative enhancer.

Supplementary Figure 5: MM 5hmC peak clusters associate with H3K27ac super-enhancers. Rank ordering of the 100 strongest $5 \mathrm{hmC}$ peak clusters in the cohort (A), in the MMSET group (B), in the CCND1 group (C) and in the hyperdiploid group (D). (E) Rank ordering of the 100 strongest super-enhancers based on the H3K27ac signal in a MM patient with genomic anomalies $t(4 ; 14) /$ del13/amp1q (patient MM2 from Jin and colleagues ${ }^{39}$ ). ( $F$ ) Rank ordering of the 100 strongest $5 \mathrm{hmC}$ clusters of a MM patient with genomic anomalies $\mathrm{t}(4 ; 14) / \mathrm{del} 13 / \mathrm{amp} 1 \mathrm{q}$ (our study: patient MM_14).

Supplementary Figure 6: $5 \mathrm{hmC}, \mathrm{ATAC}$ and H3K27ac are enriched at major MM genes. Signal tracks of $5 \mathrm{hmC}, \mathrm{ATAC}$ and H3K27ac at the loci CCND1 (A), CREB3L2 (B), DUSP22/IRF4 (C), PRDM1 (D), TCF3 and XBP1.

Supplementary Figure 7: Core transcription factors binding motifs spread over the two networks. (A) Heatmap representing the expression level of the core transcription factors in the MM cohort. (B) Network representation of the $5 \mathrm{hmC}$ peak clusters linked with the strongest correlations. Each dot is a $5 \mathrm{hmC}$ peak cluster. Edges are represented if the correlation between is 2 nodes is greater than 0,715 . Color intensity represents the normalized number of motifs ATF4, KLF6, IRF4, PRDM1 and XBP1 in the $5 \mathrm{hmC}$ peak clusters.

Supplementary Figure 8: Survival courses depending on $\mathbf{5 m C}$ and $\mathbf{5 h m C}$. (A) OS over global level of $5 \mathrm{mC}$ in MS. (B) PFS over global level of $5 \mathrm{mC}$ in MS. (C) OS over $5 \mathrm{hmC}$ enrichment at TP53INP1 genomic locus. (D) OS over the score of the signature of TP53INP1, UPF1 and RNF144A RNA expression levels (cf Methods). (Time: Number of years).

\section{Supplementary Table}

Supplementary Table: Description of the patients and genomic regions of interest. (Tab1) Patients samples information. (Tab2) Principal Component Analysis. (Tab3) Group-specific regions. (Tab4) TF network analysis. (Tab5) Diagnosis/Relapse analysis. (Tab6) Survival analysis. 


\section{Figure 1:}

A

$40 \mathrm{MM}$ diagnosis

$4 \mathrm{MM}$ paired relapse

CD138+ cells

5 Normal

purification

5hmC quantification and sequencing
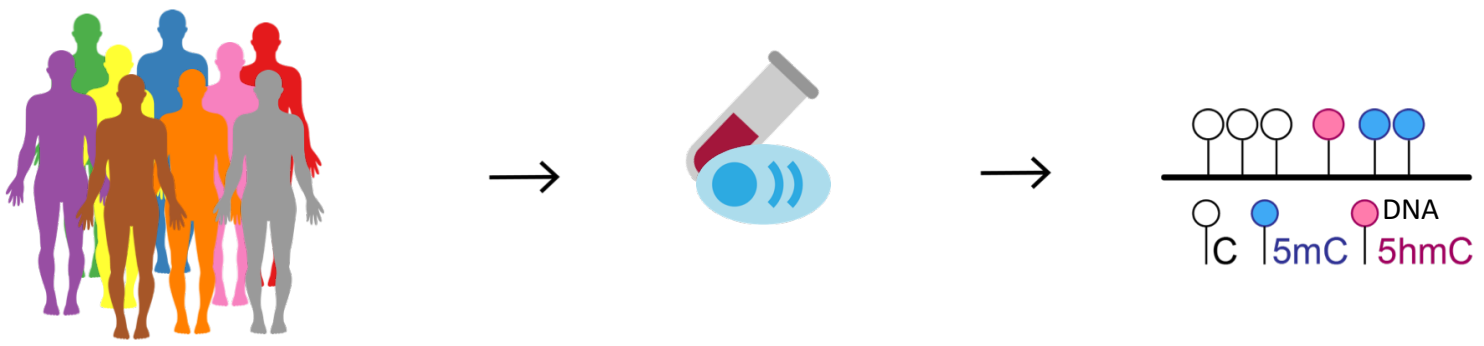

B

C
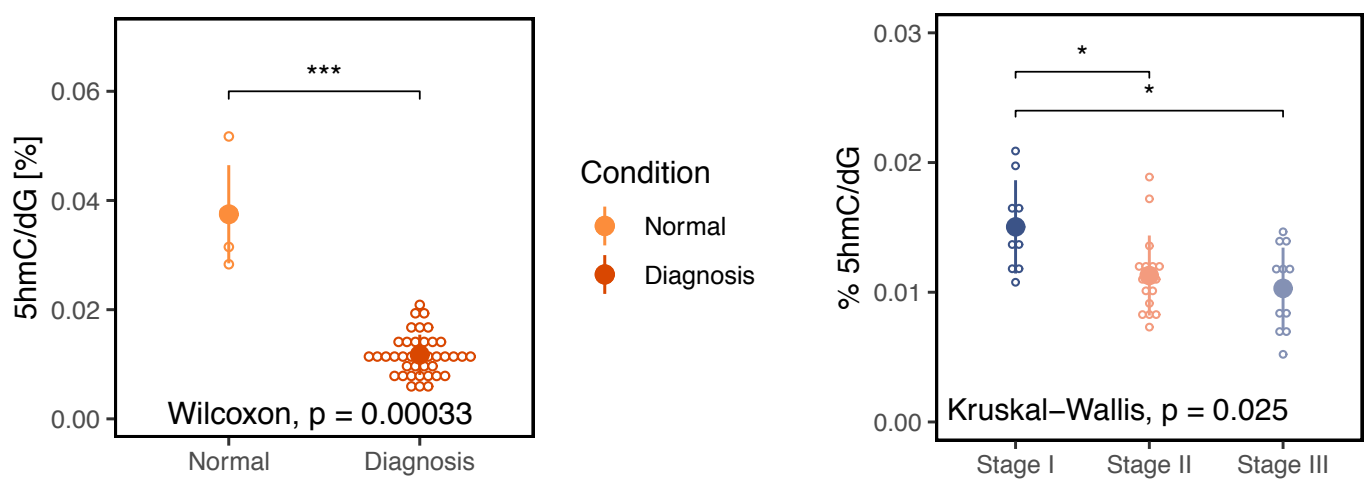

iss

Stage I

Stage II

\ Stage III

D

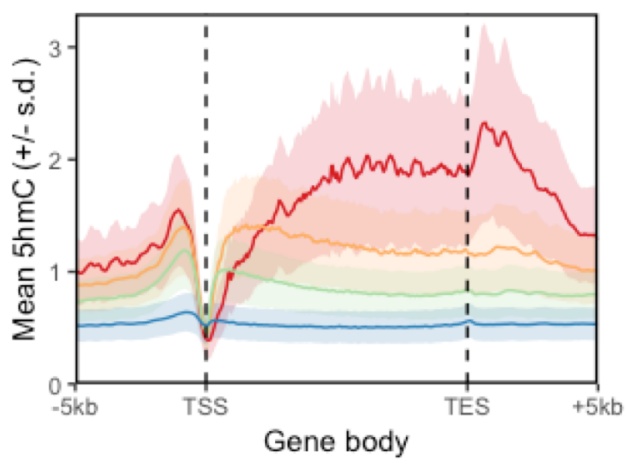

E
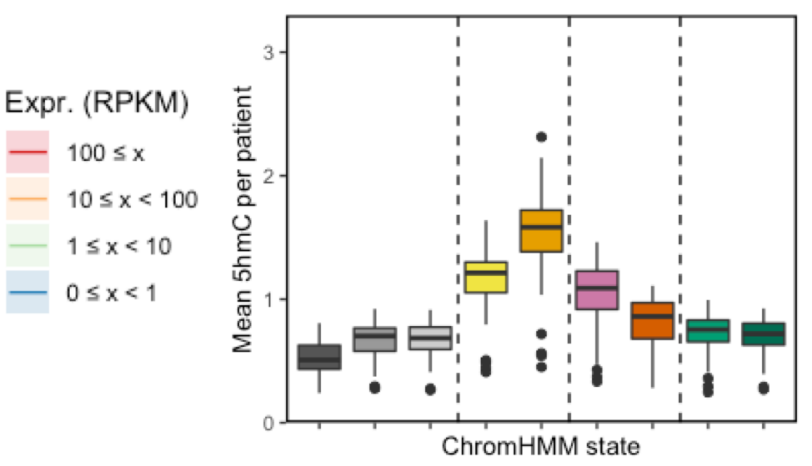

ChromHMM state

PcG Repressed

官 Heterochromatin

官 Repeats / CNV

宁 Weak Enhancer

官 Strong Enhancer

官 Weak Promoter

Strong Promoter

审 Weak Transcription

Transcription

F

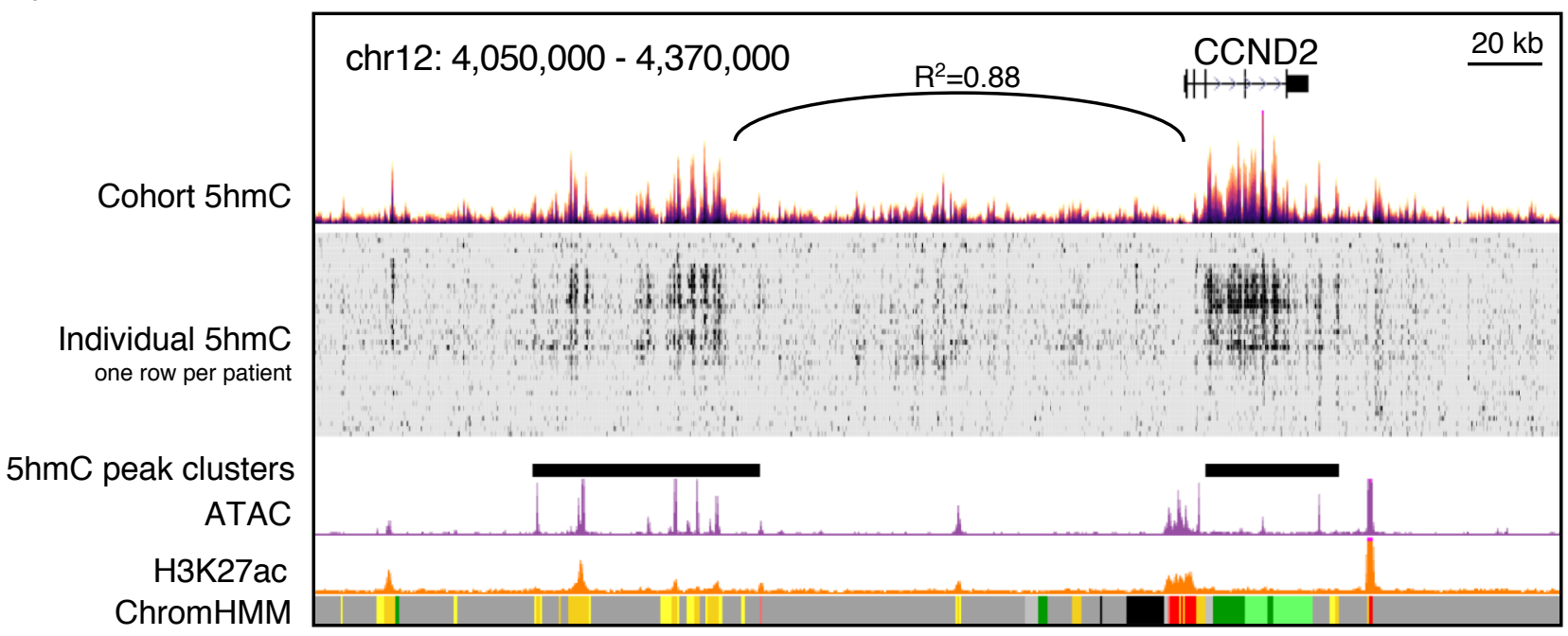


Figure 2:

A

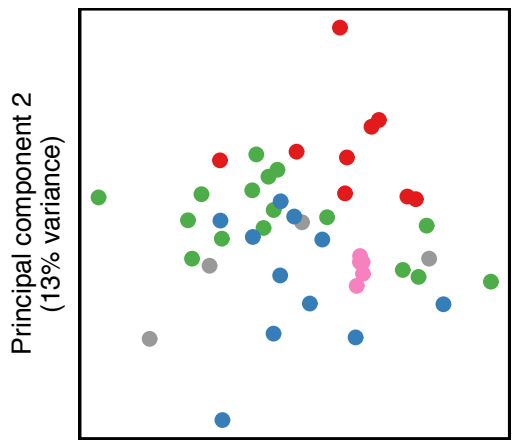

Principal component 1 (16\% variance)
B

Group

- MMSET

- CCND1

- Hyperdiploid

- Normal

- Other
C

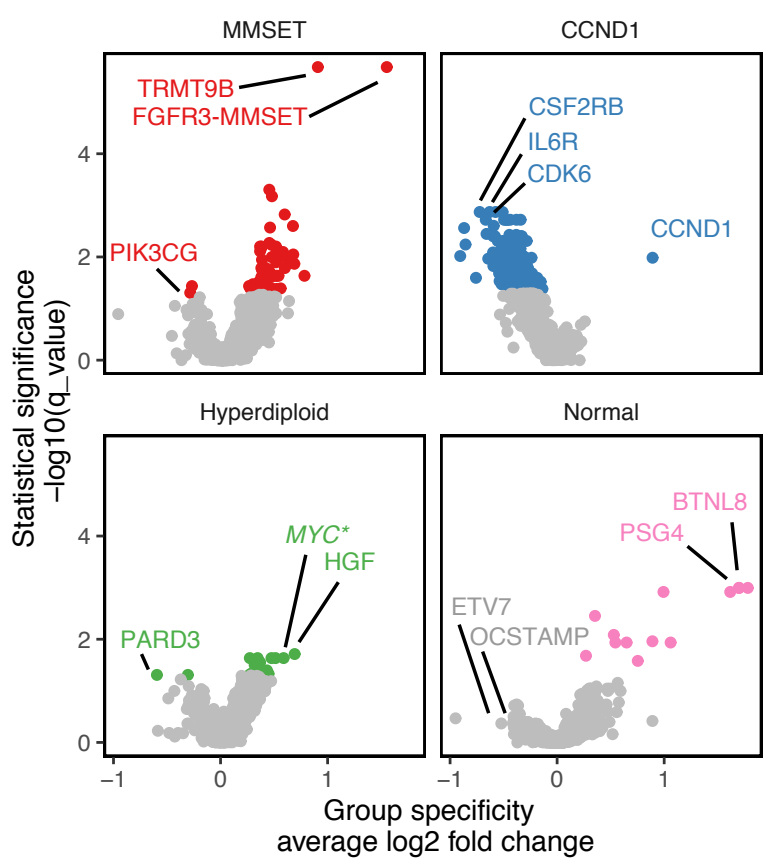

D

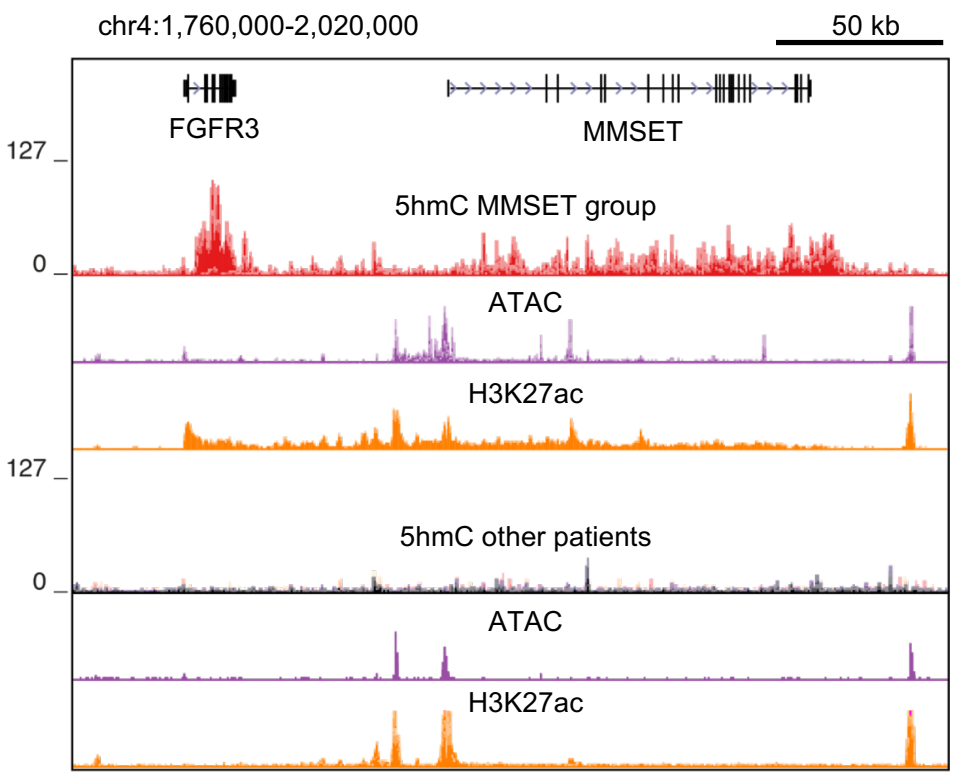

5hmC FGFR3-MMSET Fold-Difference: 5.34, adj. $p$ value $<4.2 \mathrm{E}-6$ 


\section{Figure 3:}

A

$5 \mathrm{hmC}$ correlation network clustering

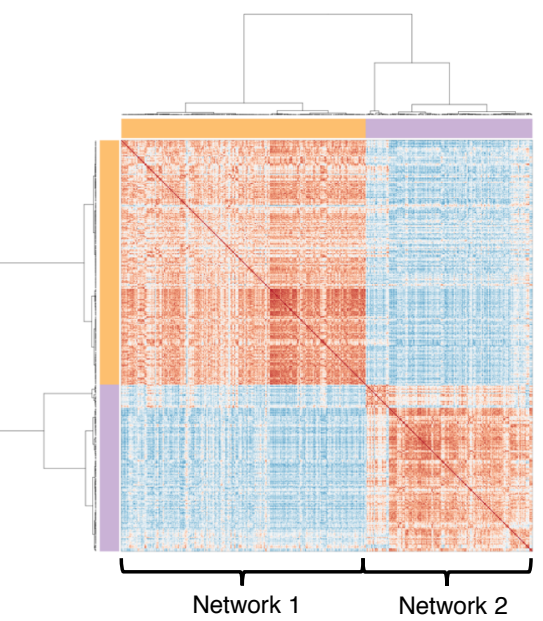

C

TCF3-MYC motifs in $5 \mathrm{hmC}$ regions

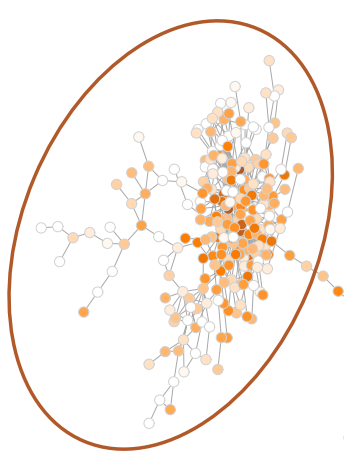

Network 1

(TCF3-MYC enriched)

Network 2

$E$

IRF4-PRDM1 motifs in 5 $\mathrm{hmC}$ regions

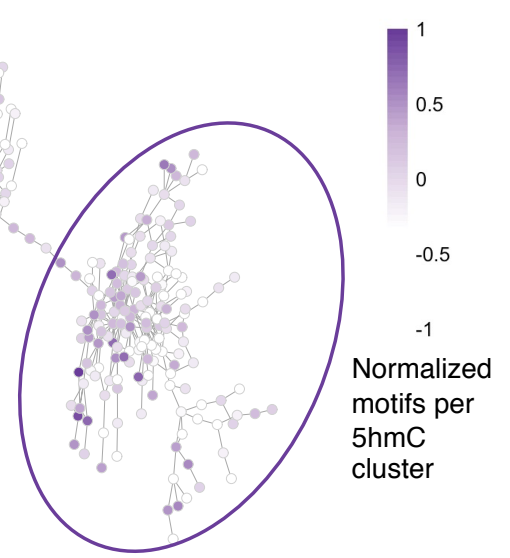

Network 2

(IFR4-PRDM1 enriched)

B

$\mathrm{N}=407$

$5 \mathrm{hmC}$ peak clusters

D

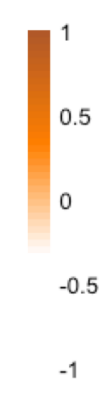

Normalized motifs per $5 \mathrm{hmC}$ cluster
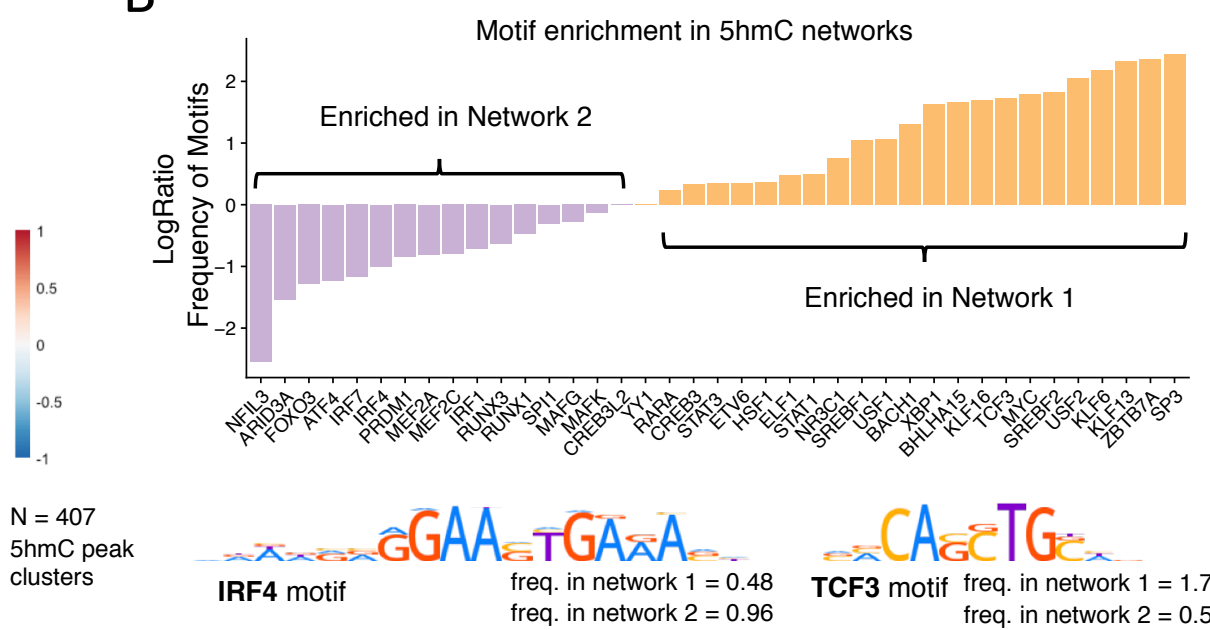

TCF3 motif freq. in network $1=1.7$ freq. in network $2=0.51$

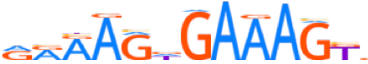

PRDM1 motif

freq. in network $1=0.42$

freq. in network $2=0.76$

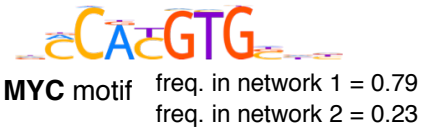

MYC motif freq. in network $1=0.79$
freq in network $2=0.23$

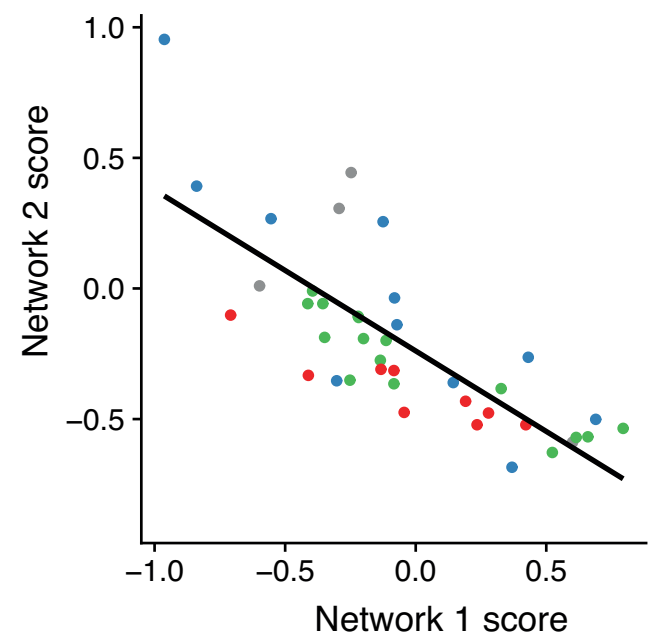

group

- CCND1

- $\mathrm{HD}$

- MMSET

- Unknown

Coef $=-0.62$ $R^{2}=0.60$
F

\begin{tabular}{|c|c|c|c|c|}
\hline $\begin{array}{l}\text { GREAT V3 } \\
\text { ANALYSIS }\end{array}$ & GO Biological Process & $\begin{array}{c}\text { Fold } \\
\text { Enrichment }\end{array}$ & $\begin{array}{l}\text { Binomial FDR } \\
\text { q-value }\end{array}$ & Associated expressed genes \\
\hline \multirow{2}{*}{ Network 1} & $\begin{array}{l}\text { negative regulation of } \\
\text { cytokine production }\end{array}$ & 6.0367 & $3.4972 \mathrm{e}-40$ & $\begin{array}{l}\text { CACTIN, GAS6, HSF1, JAK3, } \\
\text { KLF2, NLRC5, RAC1, RARA, } \\
\text { RGCC, TGFB1, TRAF3, TSPO }\end{array}$ \\
\hline & $\begin{array}{l}\text { negative regulation of } \\
\text { tumor necrosis factor } \\
\text { production }\end{array}$ & 13.7173 & $1.4718 \mathrm{e}-35$ & $\begin{array}{l}\text { CACTIN, GAS6, HSF1, RARA, } \\
\text { TSPO }\end{array}$ \\
\hline \multirow{2}{*}{ Network 2} & $\begin{array}{l}\text { immune response- } \\
\text { regulating cell surface } \\
\text { receptor signaling } \\
\text { pathway }\end{array}$ & 4.5929 & $4.0779 \mathrm{e}-44$ & $\begin{array}{l}\text { BTLA, DUSP6, ELMO1, FGFR3, } \\
\text { FOXO3, GAB1, IRAK2, MEF2A, } \\
\text { PDE4B, PPP3CA, PRKD2, SRC, } \\
\text { ZAP70 }\end{array}$ \\
\hline & $\begin{array}{l}\text { immune response- } \\
\text { regulating signaling } \\
\text { pathway }\end{array}$ & 3.9194 & $2.4855 e-40$ & $\begin{array}{l}\text { BTLA, ELMO1, FGFR3, FOXO3, } \\
\text { GAB1, MEF2A, PPP3CA, } \\
\text { PRKD2, SRC, ZAP70 }\end{array}$ \\
\hline
\end{tabular}




\section{Figure 4:}

A

Patient MM_2

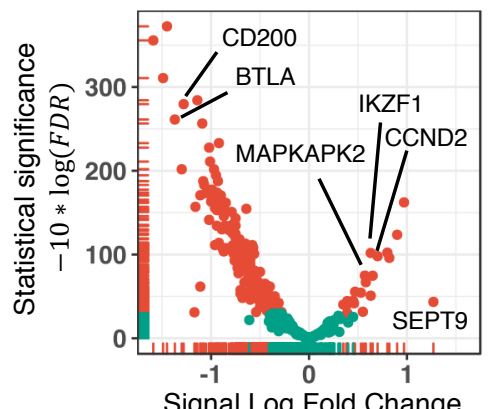

Patient MM_5

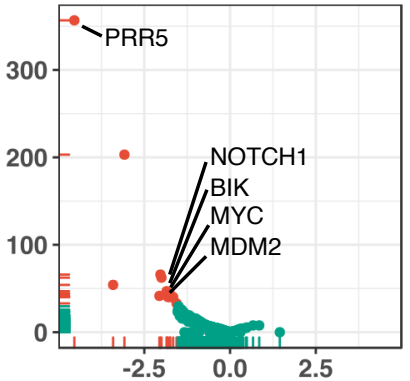

Patient MM_7

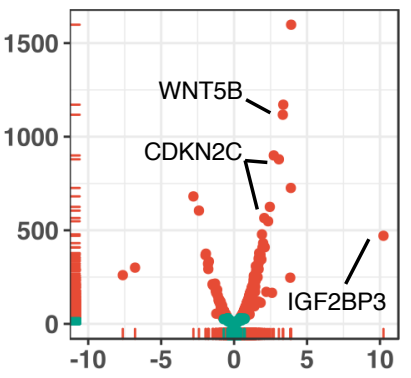

Patient MM_21

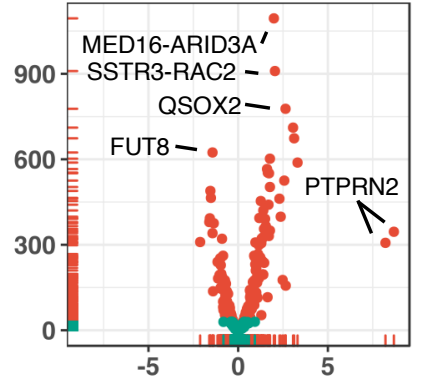

Higher at diagnosis Higher at relapse

B

\begin{tabular}{|c|c|c|}
\hline Patient & Sample & $\begin{array}{l}\text { CNV and } \\
\text { translocations }\end{array}$ \\
\hline \multirow{2}{*}{ MM_2 } & Diagnosis & $\mathrm{t}(4 ; 14) ; \operatorname{del}(1 p, 13,17 q)$ \\
\hline & Relapse & $+a m p(1 q)$ \\
\hline \multirow{2}{*}{ MM_5 } & Diagnosis & $\operatorname{amp}(3,9,11,15,19)$ \\
\hline & Relapse & $+\operatorname{del}(17 p)($ TP53) \\
\hline \multirow{2}{*}{ MM_7 } & Diagnosis & $\begin{array}{l}\mathrm{t}(4 ; 14) ; \operatorname{amp}(1 \mathrm{q}) ; \\
\operatorname{del}(13,17)\end{array}$ \\
\hline & Relapse & $+\operatorname{del}(3 p, 6 q, 8 q)$ \\
\hline \multirow{2}{*}{ MM_21 } & Diagnosis & $\begin{array}{l}\text { amp }(1 q, 3 p, 6,7,15,17 \\
19 p) ; \text { del(1p,13); } \\
\text { del(TET2) }\end{array}$ \\
\hline & Relapse & $\begin{array}{l}\text { TET2 two } \\
\text { heterozygous copies }\end{array}$ \\
\hline
\end{tabular}

C

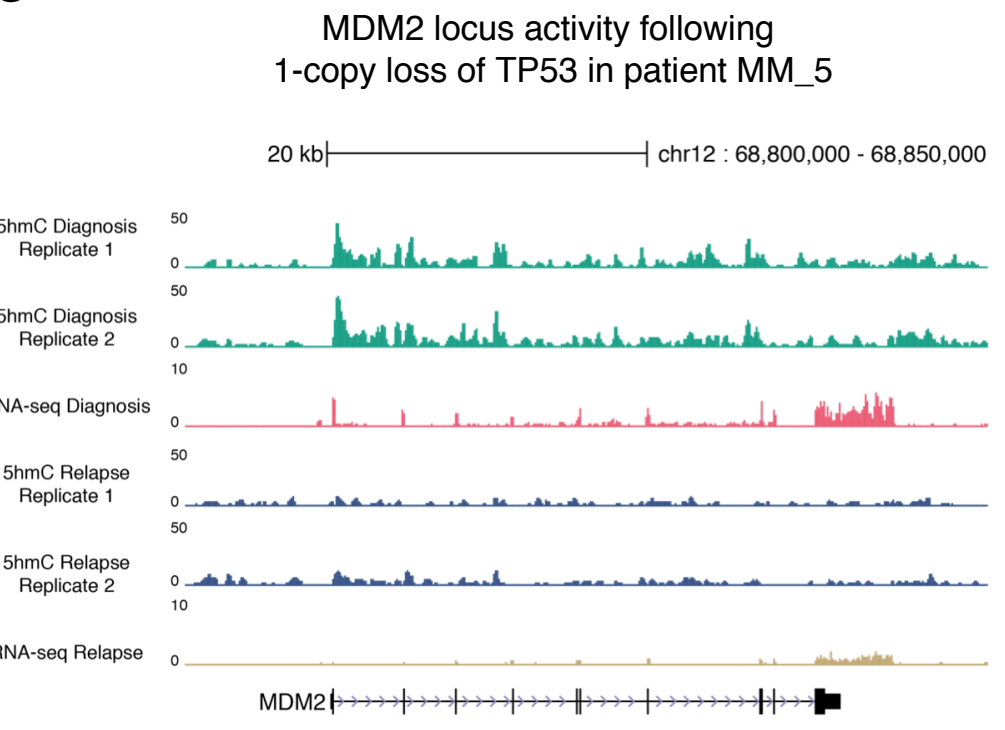




\section{Figure 5:}

A

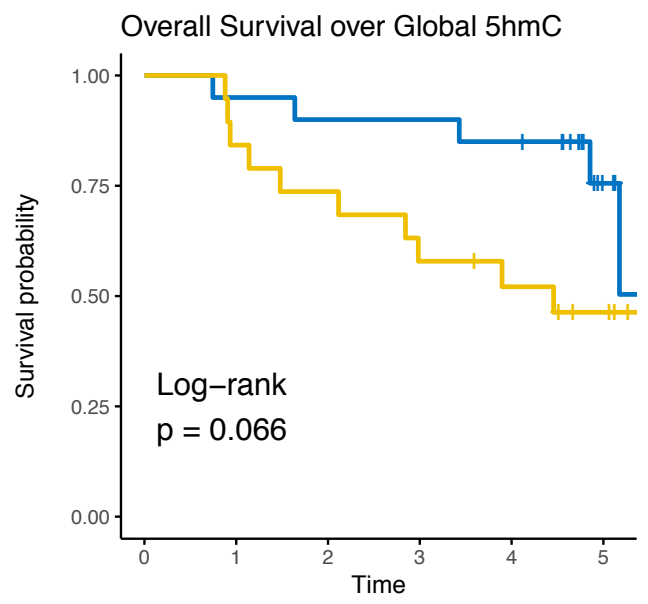

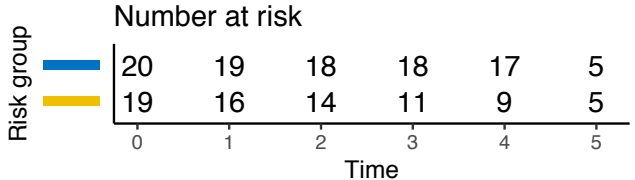

C Overall Survival over 5hmC Signature in 3 Regions

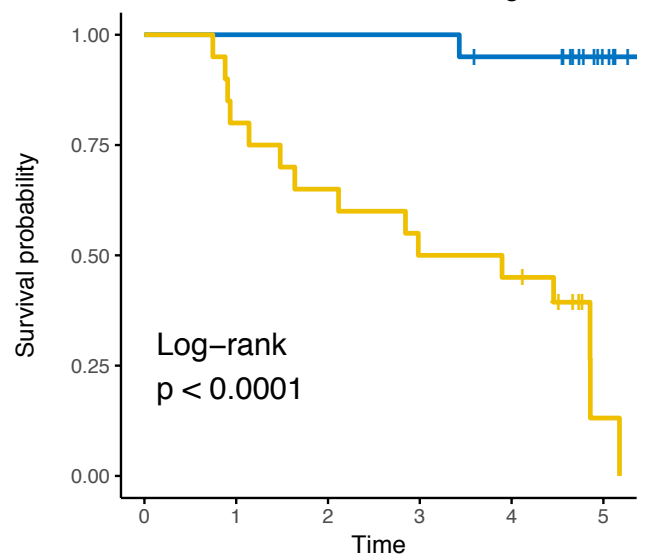

Number at risk

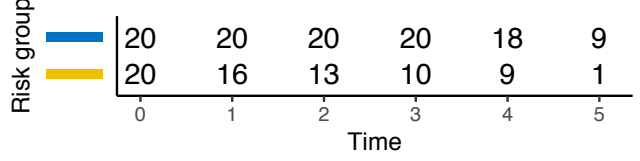

E

Overall Survival over $5 \mathrm{hmC}$ Signature in 3 Regions + Global Level

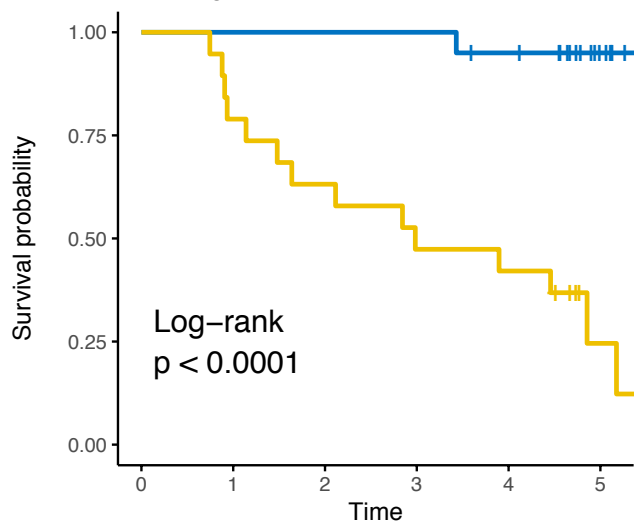

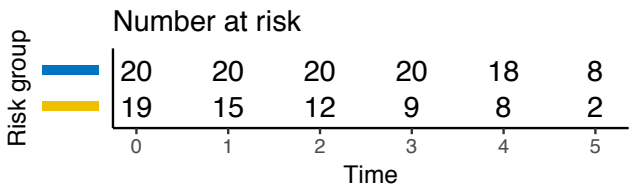

B

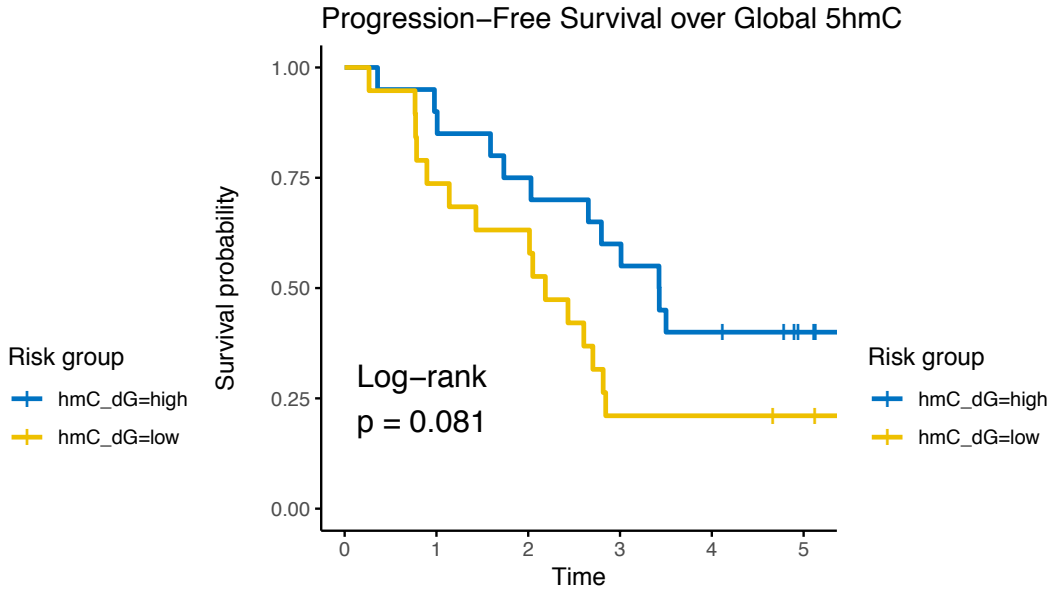

Progression-Free Survival over $5 \mathrm{hmC}$ Signature in 3 Regions

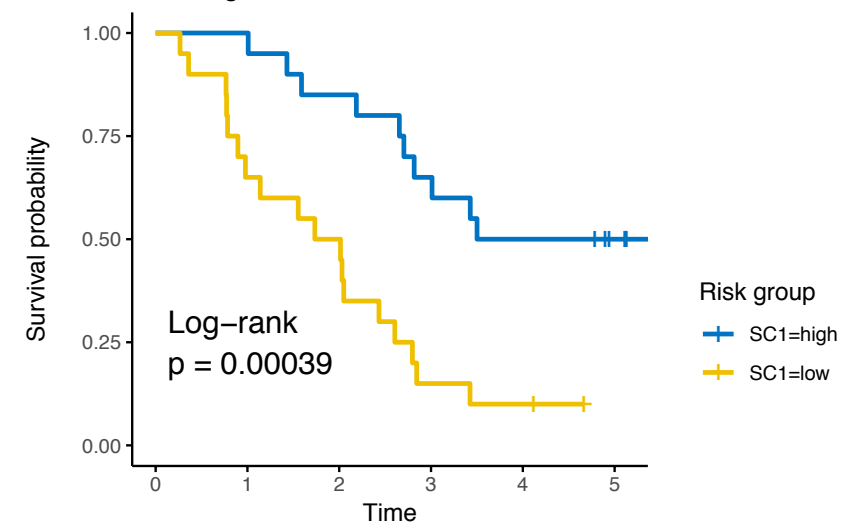

F $\quad$ Progression-Free Survival over $5 \mathrm{hmC}$ Signature

Risk group

$+\mathrm{SC} 1=$ high

$+\mathrm{SC} 1=$ low
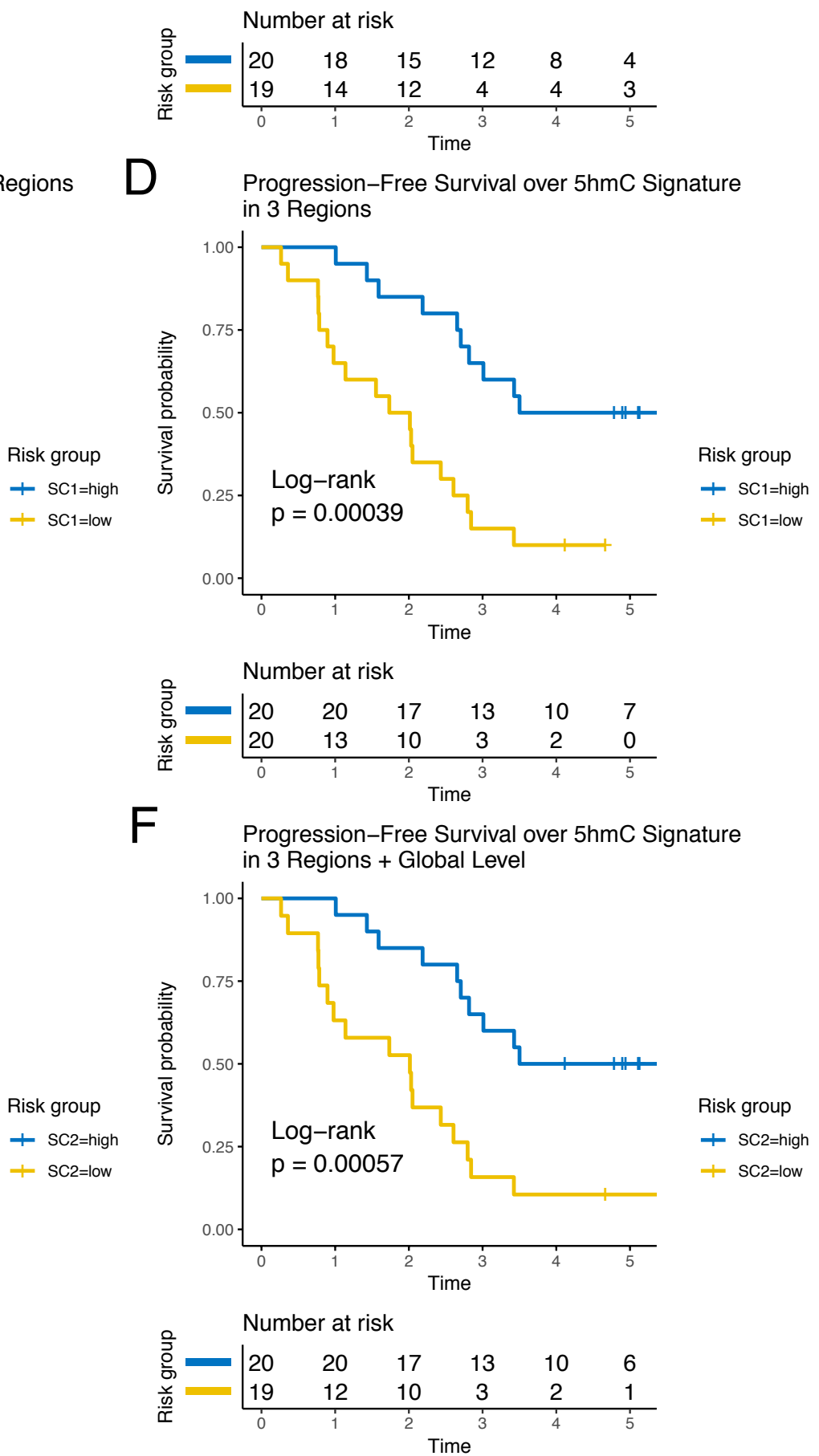


\section{Supplementary Figure 1:}
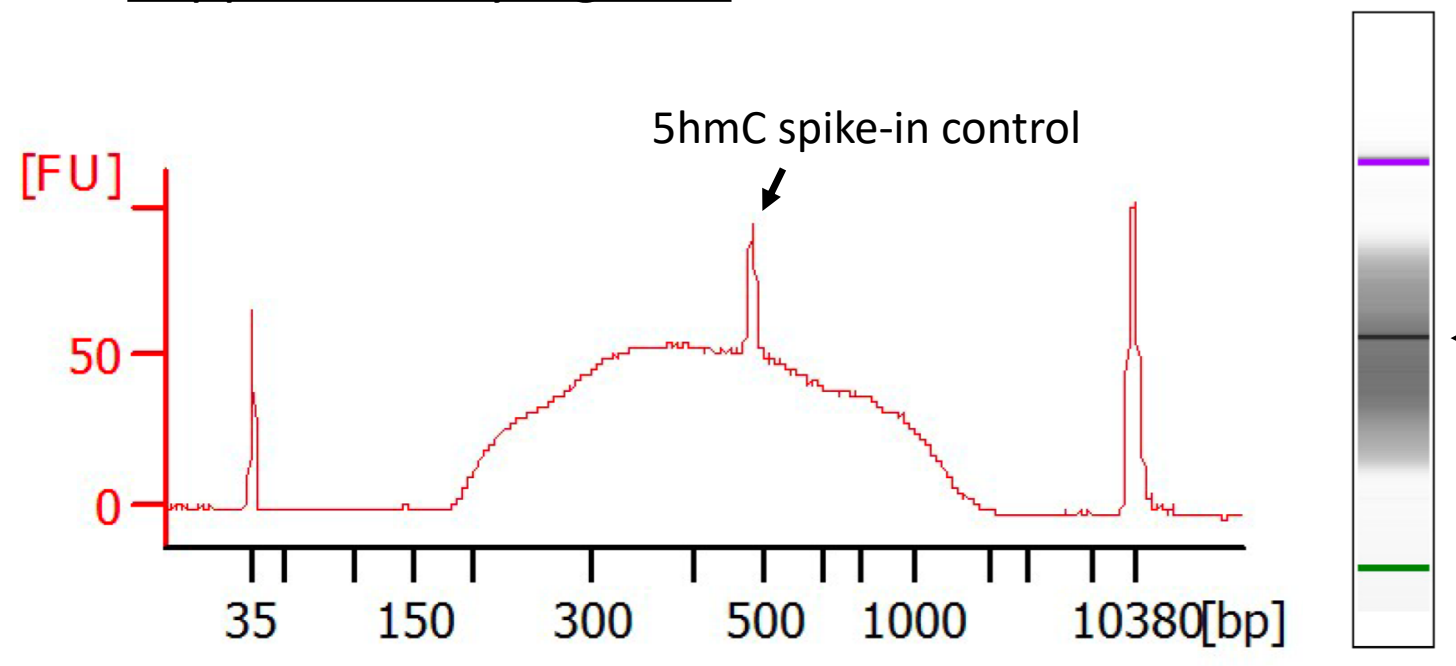

10380 bp marker

$\leftarrow$ hhmC spike-in control

35 bp marker 
Supplementary Figure 2:

Proportion of peaks remaining for each patient and median value

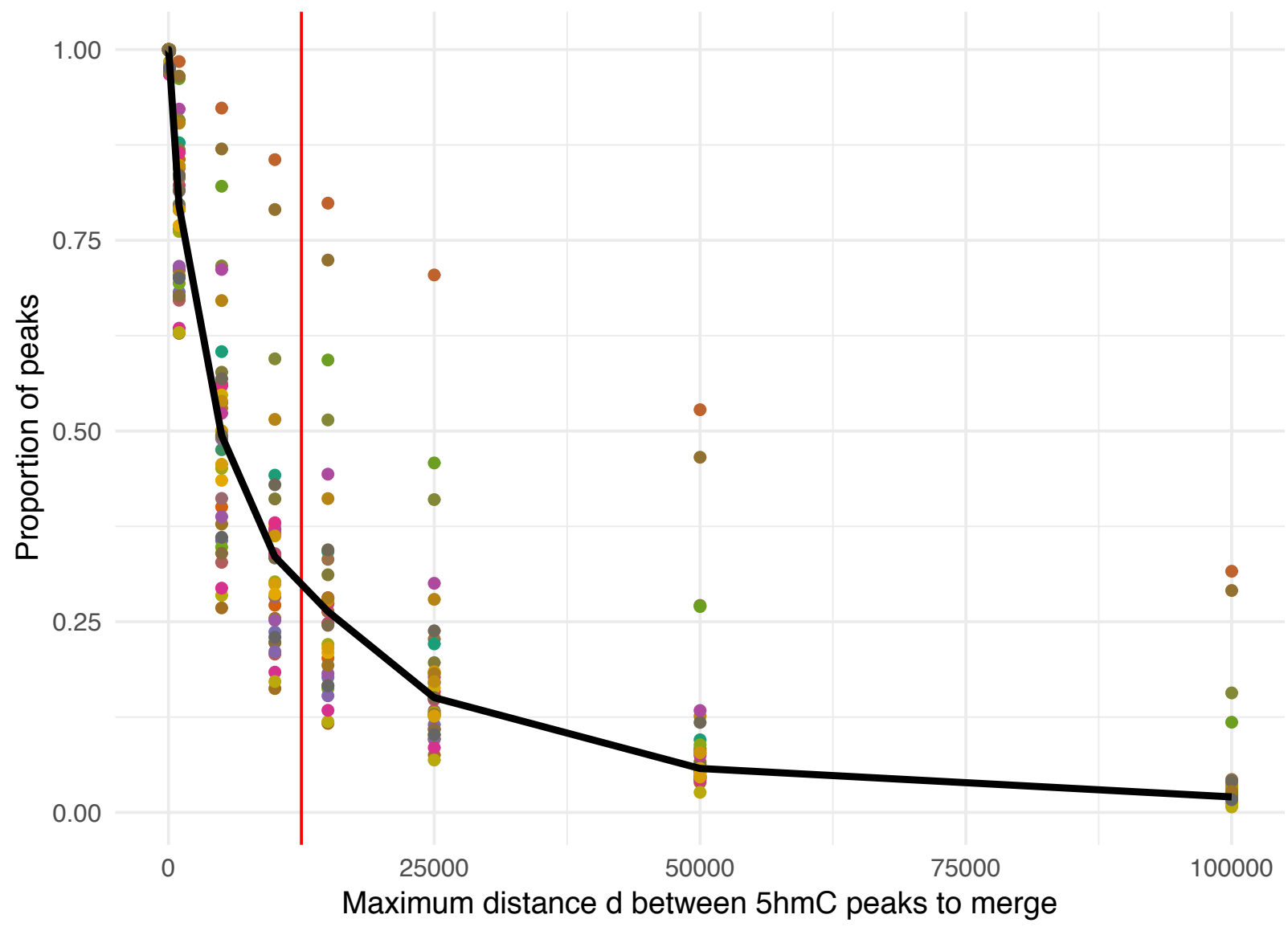




\section{Supplementary Figure 3:}

A

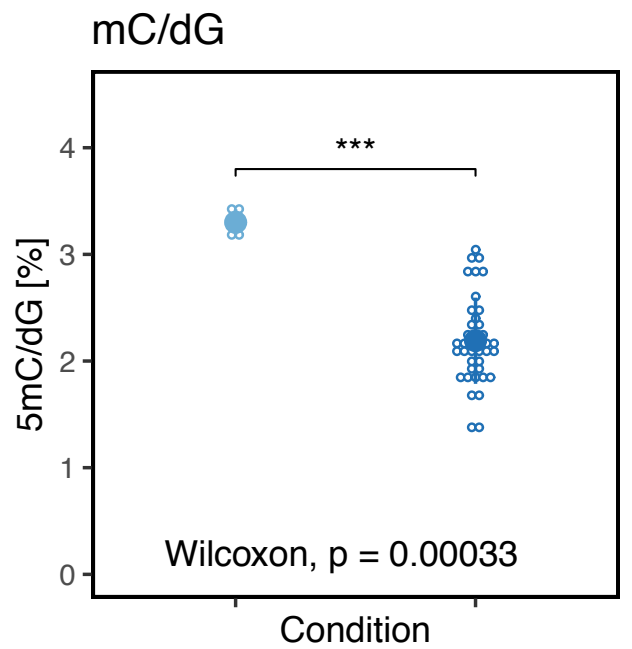

C

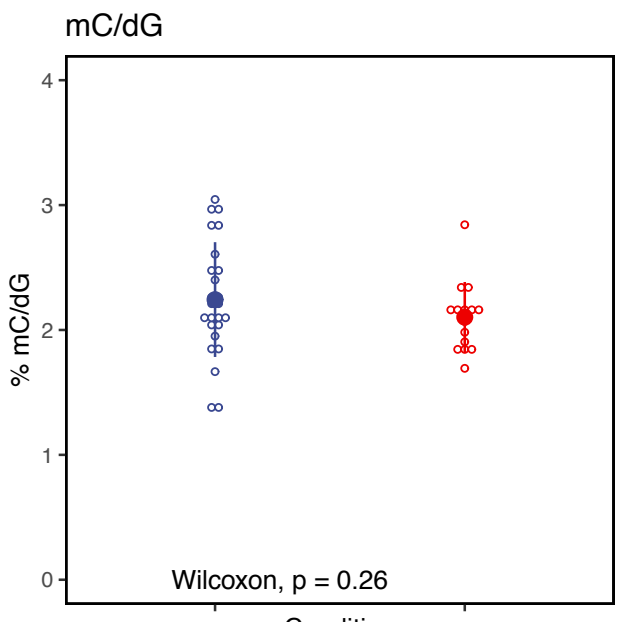

E

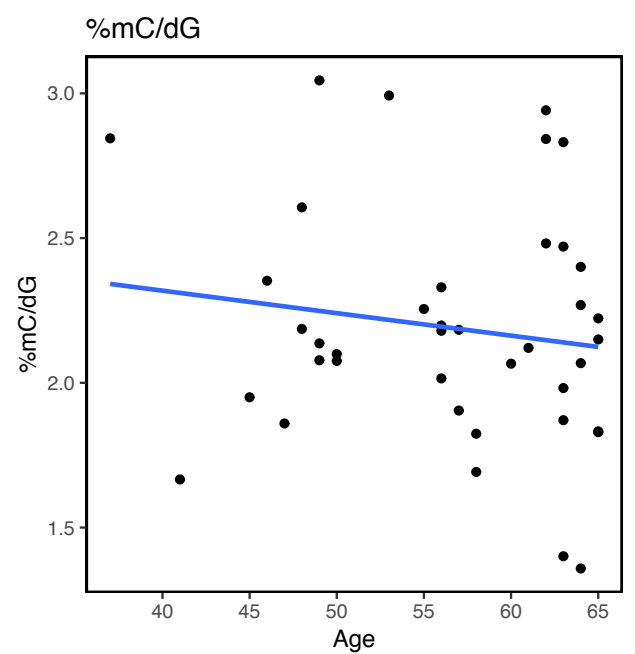

B

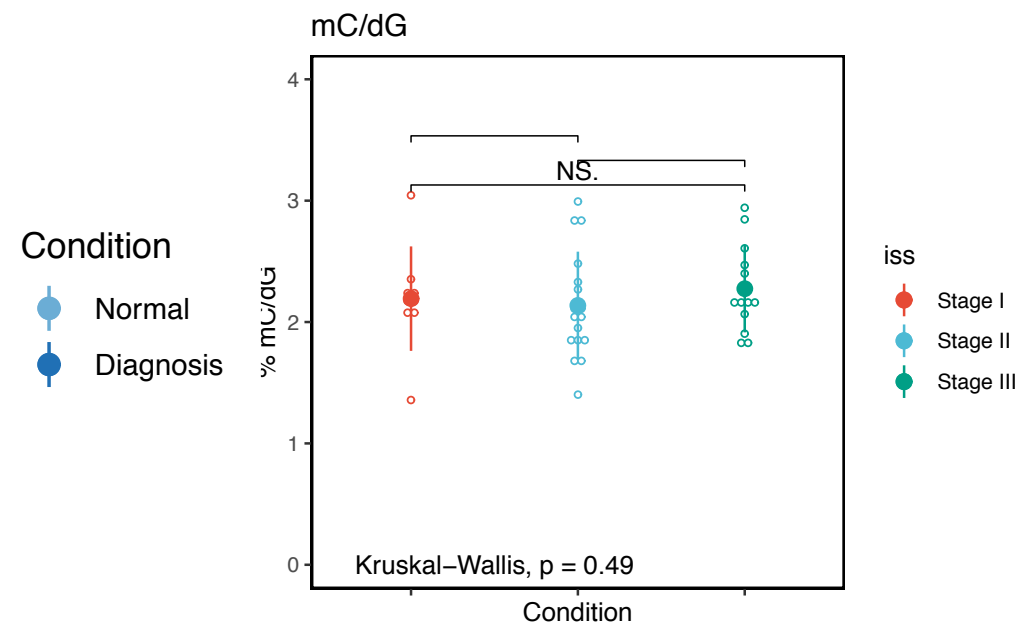

D

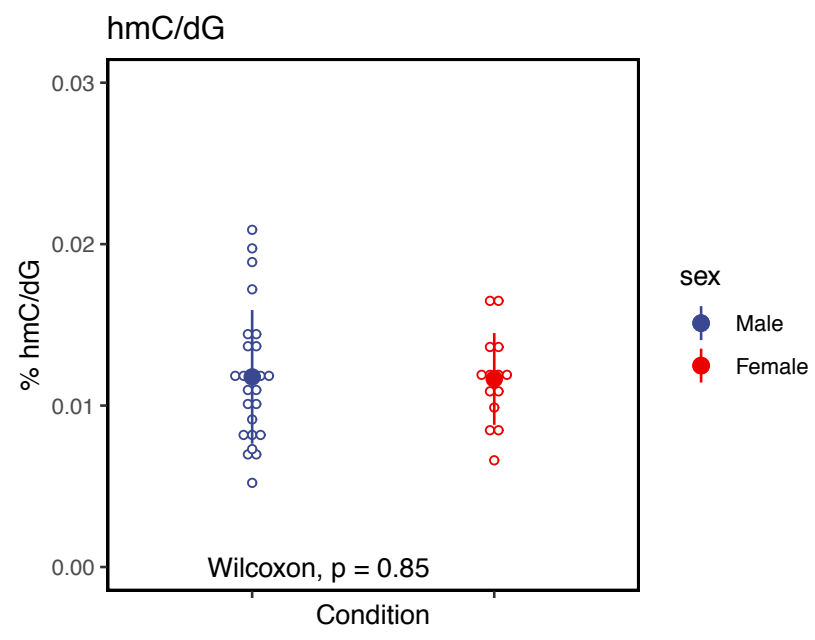

F

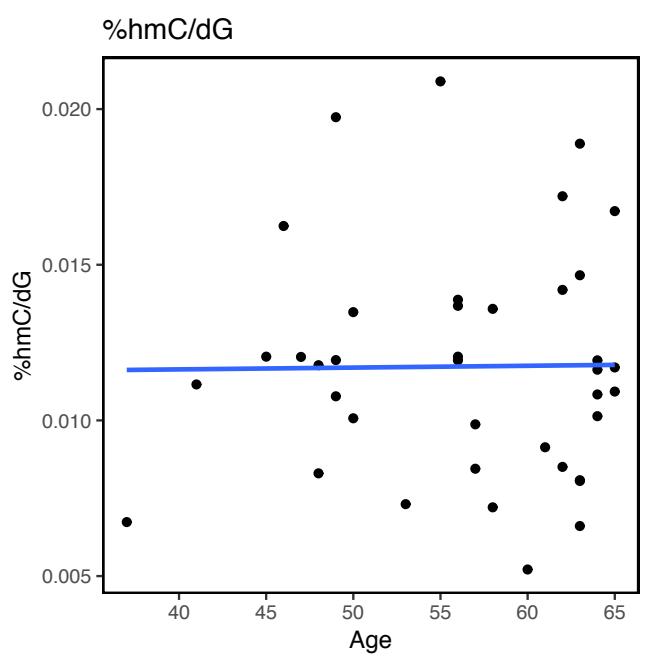




\section{Supplementary Figure 4:}

A

$5 \mathrm{hmC}$ at enhancer versus CCND2 expr.

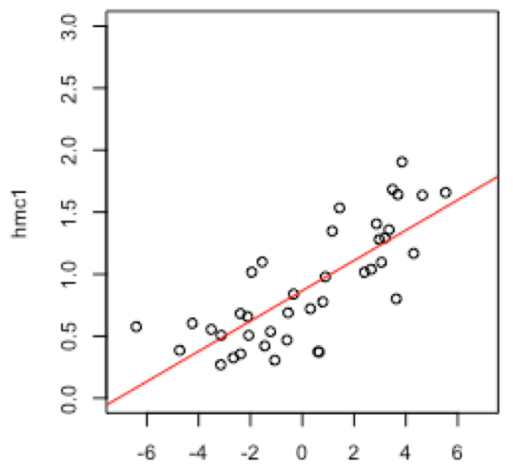

expr

adj $R^{\wedge} 2: 0.59$
$5 \mathrm{hmC}$ at gene versus CCND2 expr.

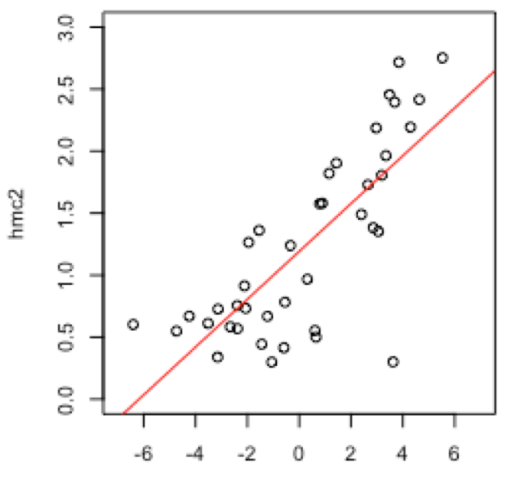

adj $R^{\wedge} 2: 0.58$
$5 \mathrm{hmC}$ at gene versus $5 \mathrm{hmC}$ at enhancer

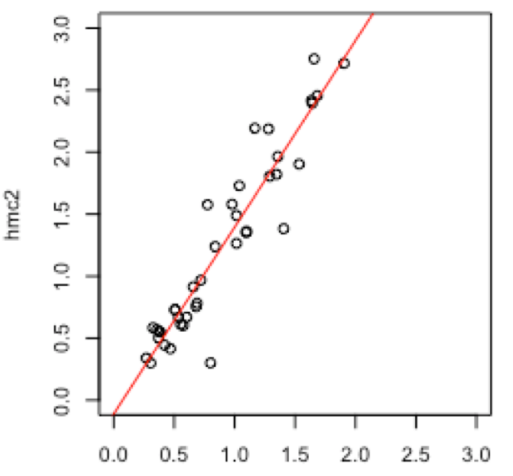

hmc1

adj $R^{\wedge} 2: 0.88$

B

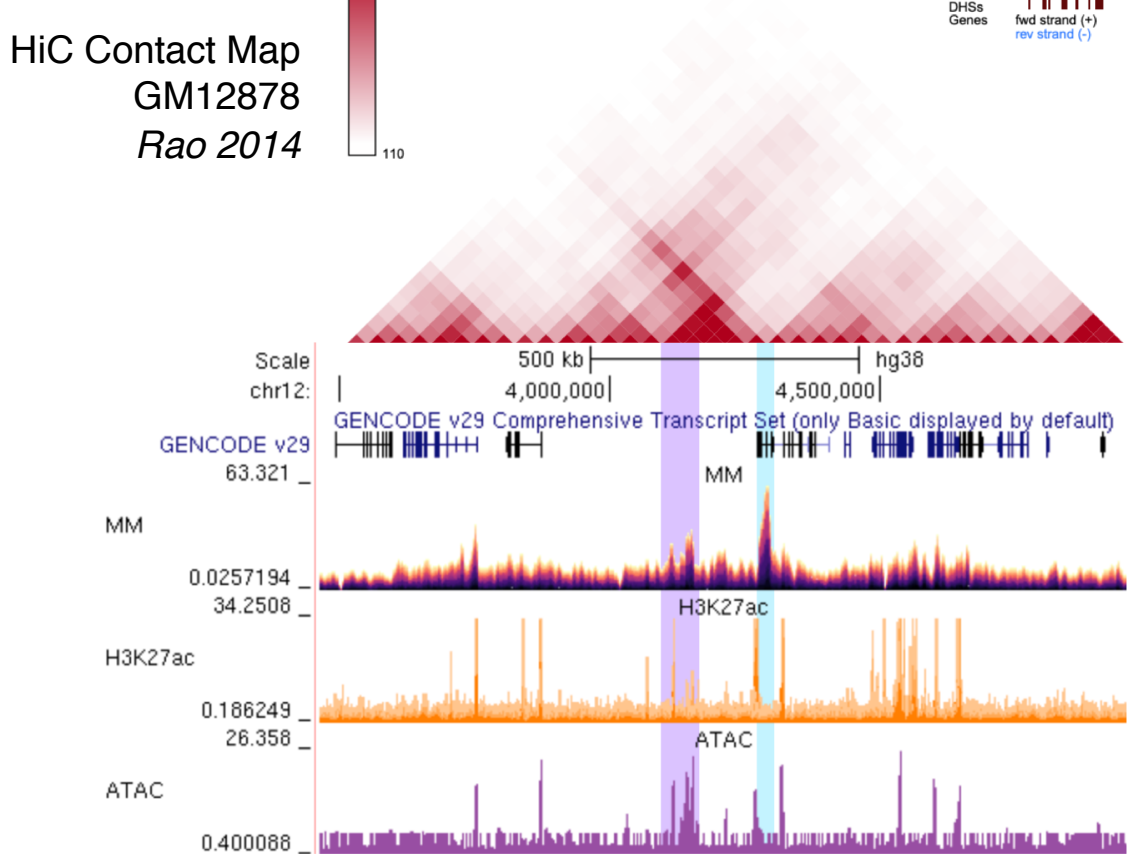




\section{Supplementary Figure 5:}

A

Whole cohort

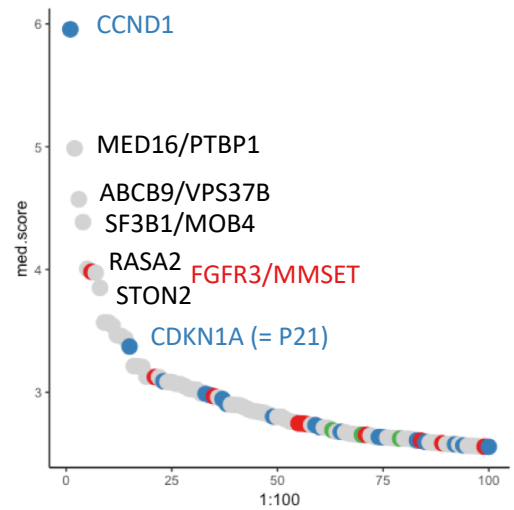

C

CCND1 group

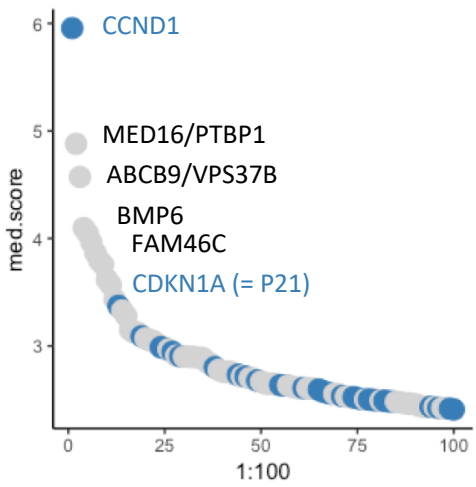

$E$

H3K27ac

(patient MM2, Jin et al. 2018)

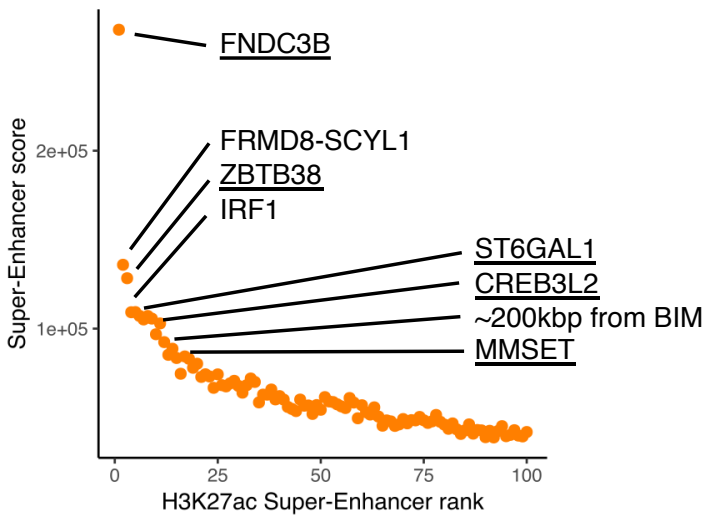

B

MMSET group

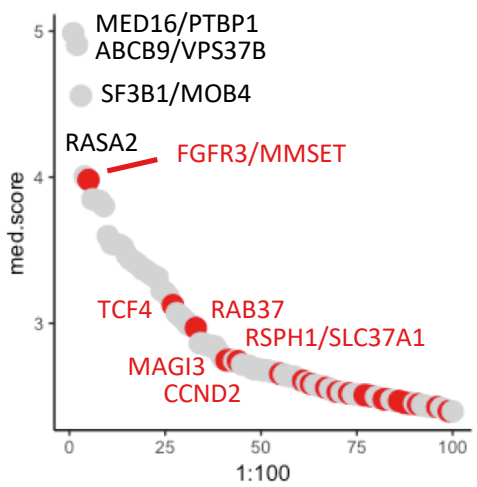

D Hyperdiploid group

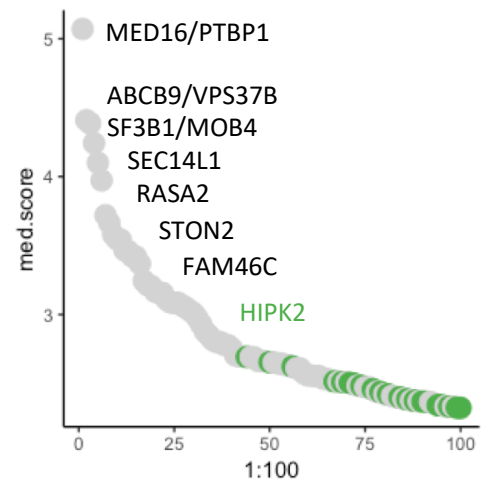

F $5 \mathrm{hmC}$ (patient MM_14)

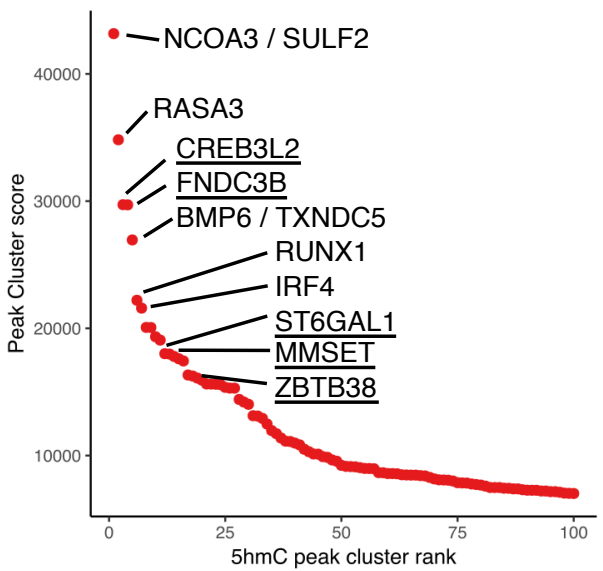




\section{Supplementary Figure 6:}

A

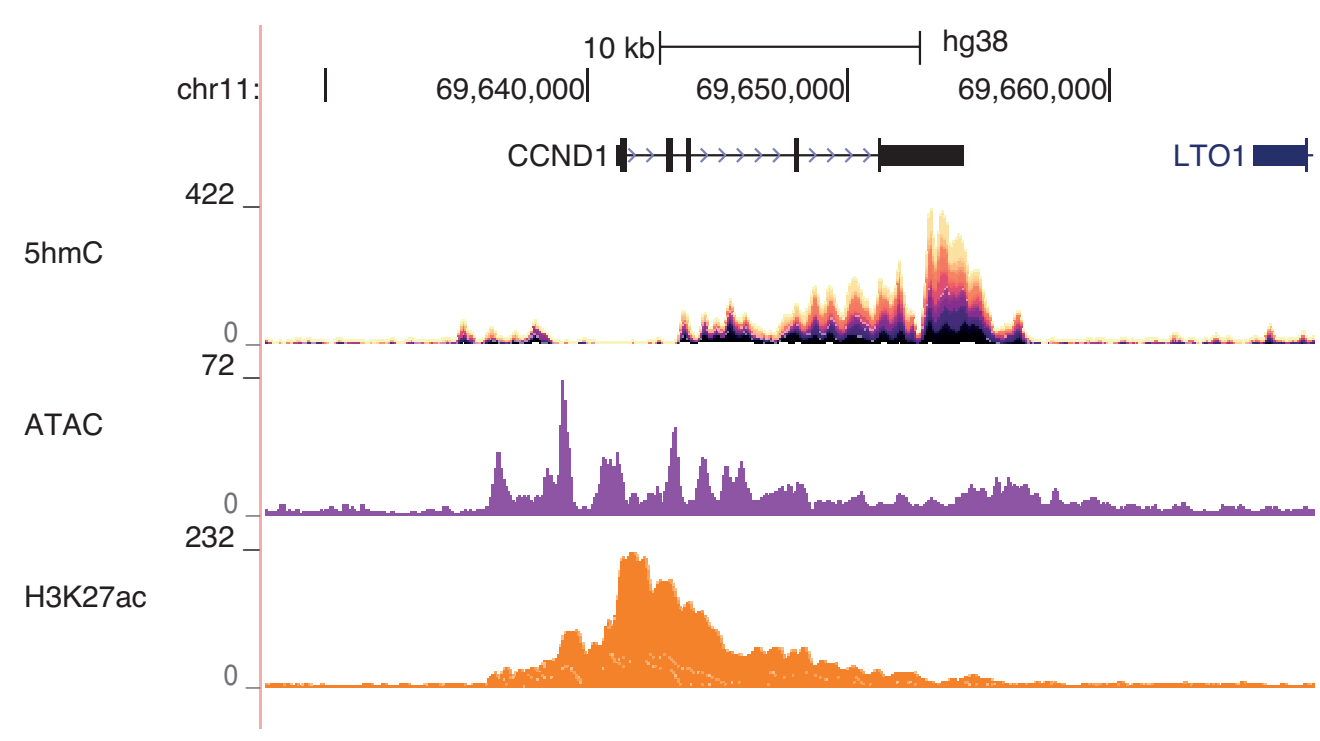

B
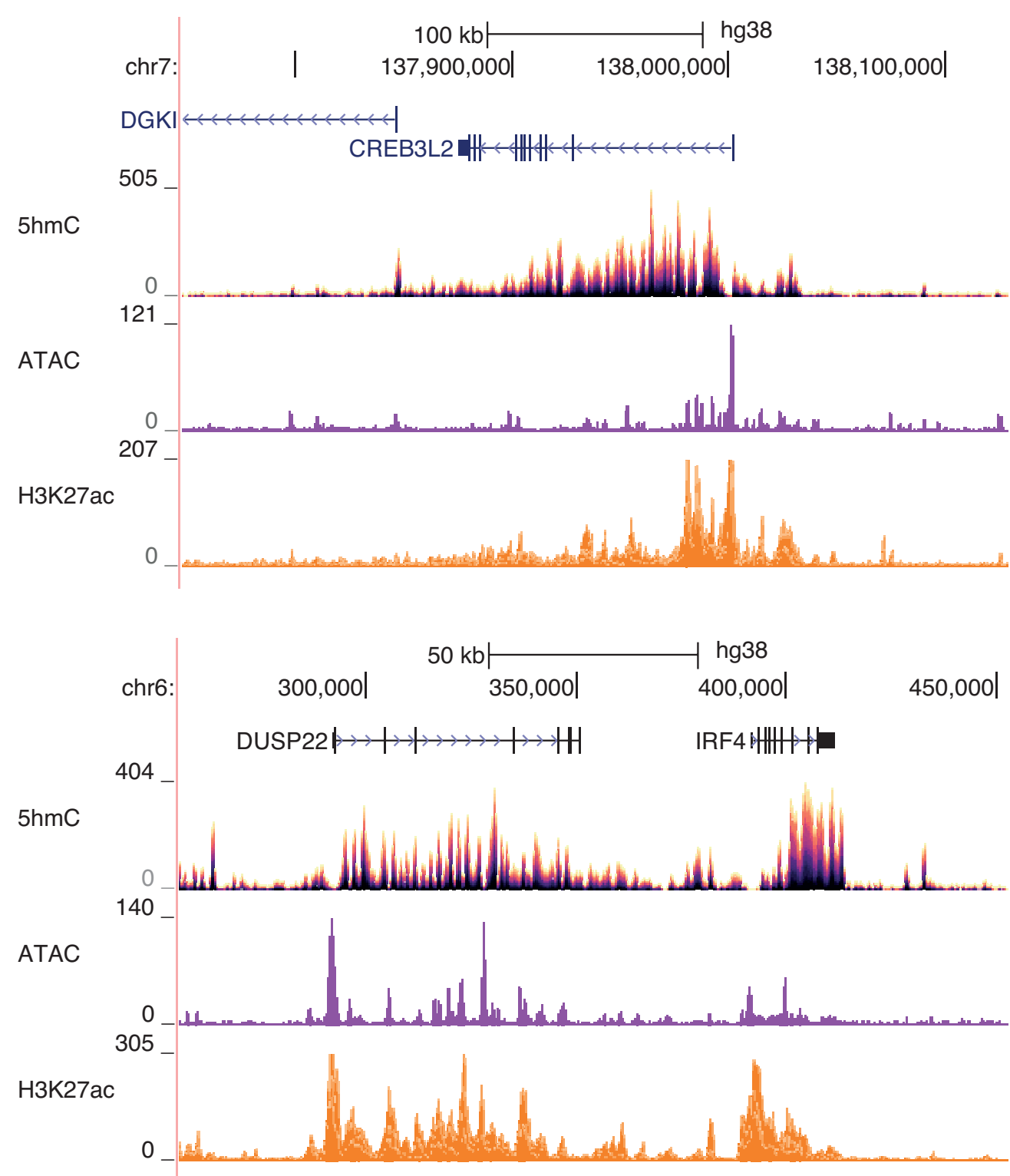
Supplementary Figure 6 (Continued):

D

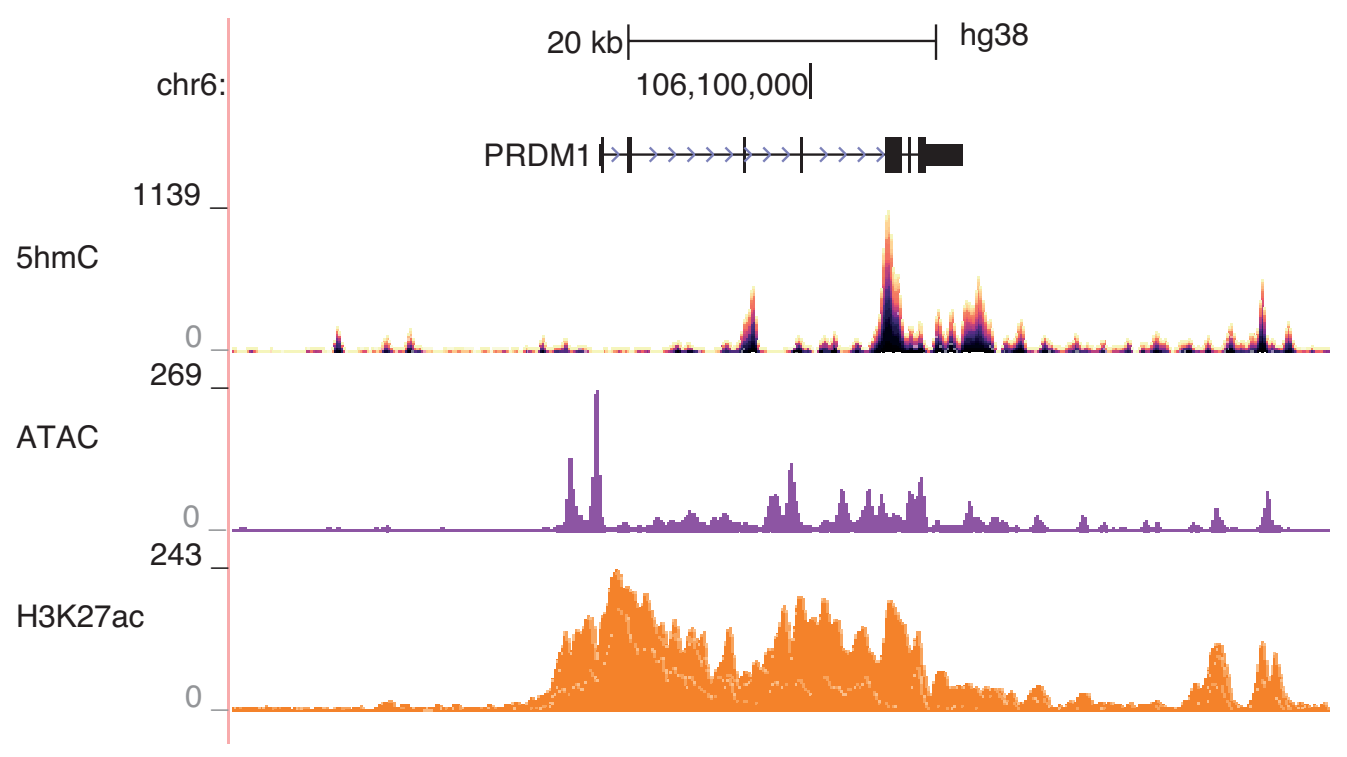

E

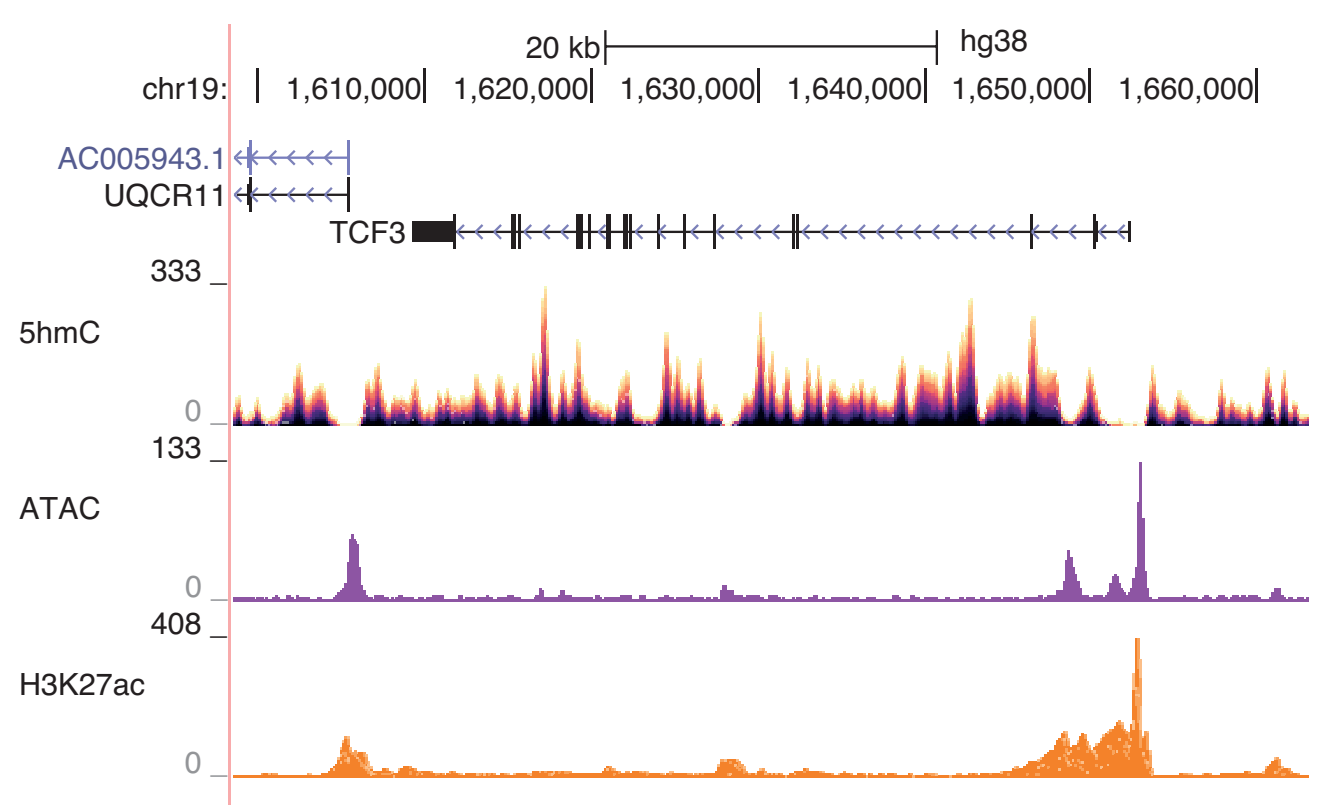

F

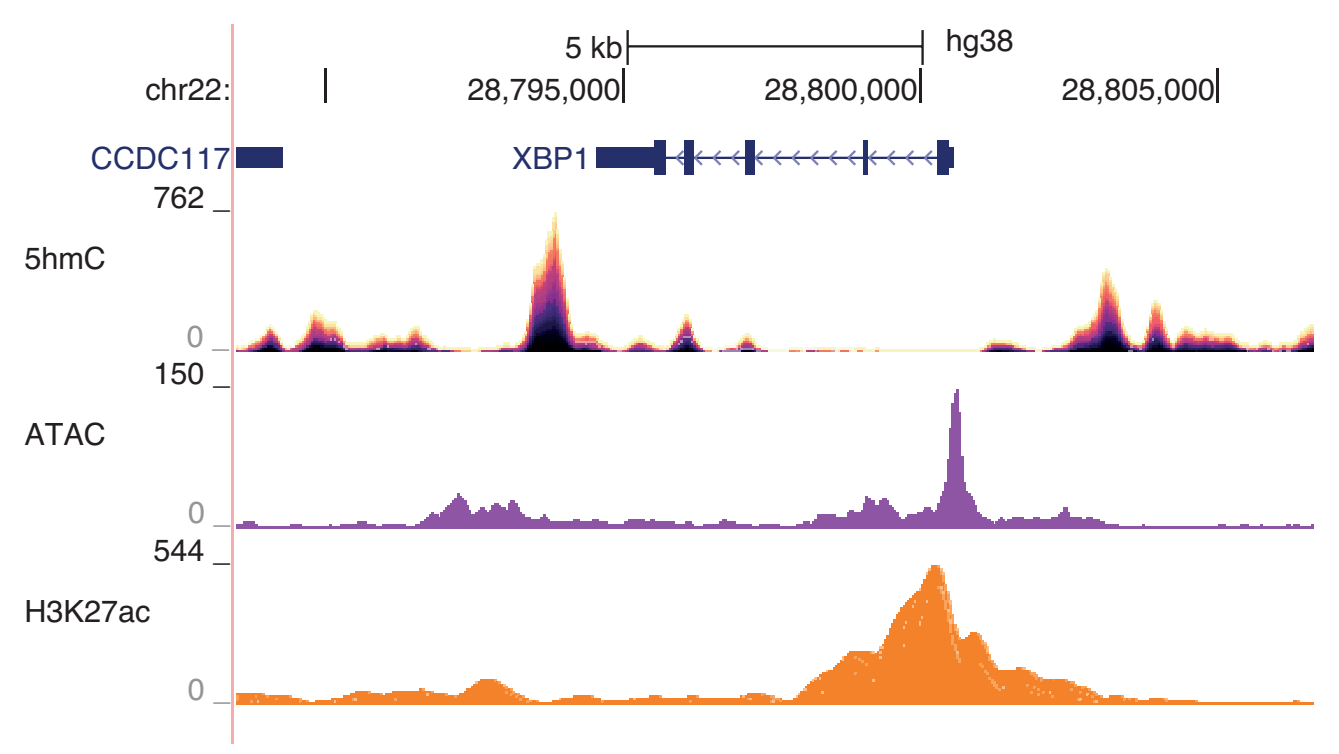




\section{Supplementary Figure 7:}

A

Core Transcription Factors Expression

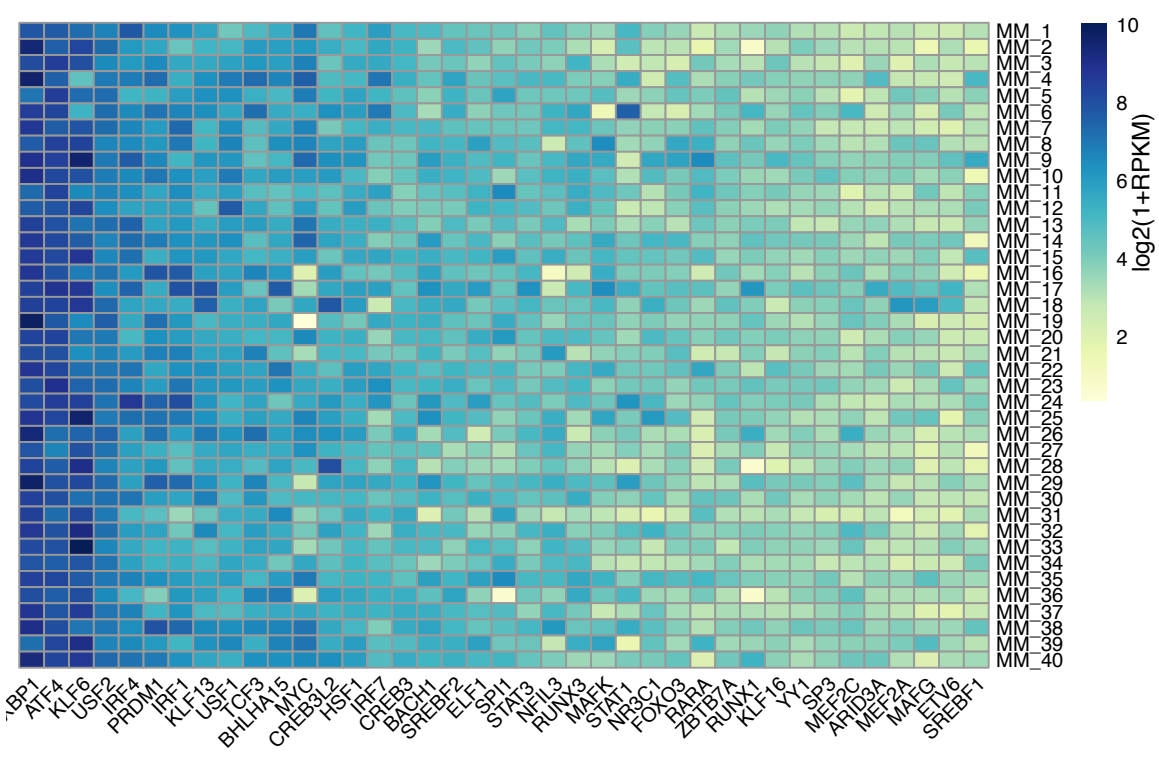

B

ATF4

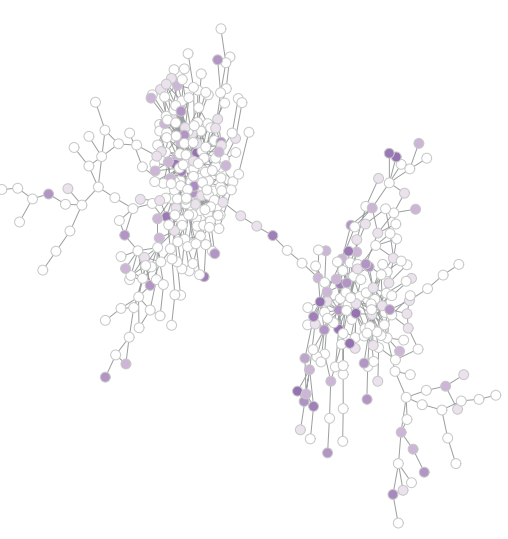

IRF4

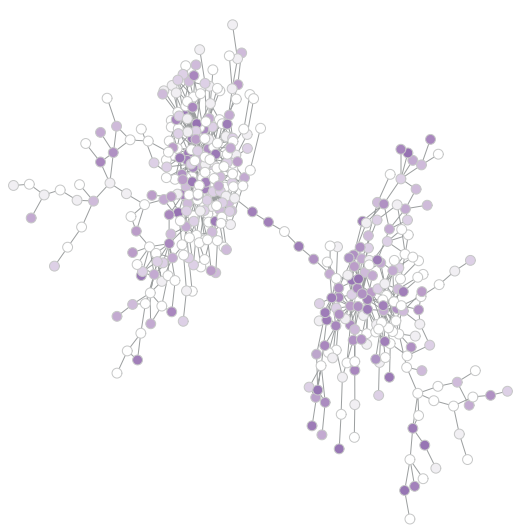

PRDM1

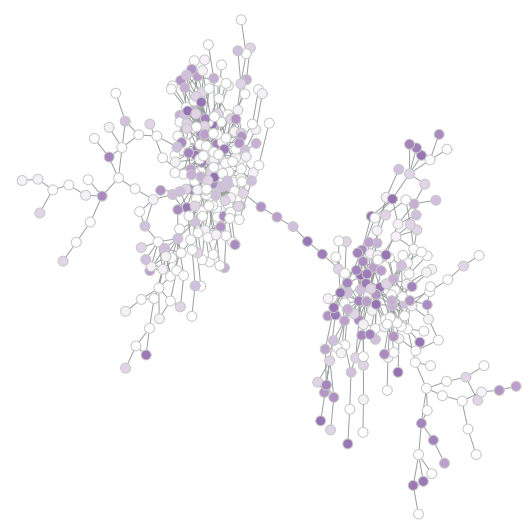

\section{KLF6}

XBP1
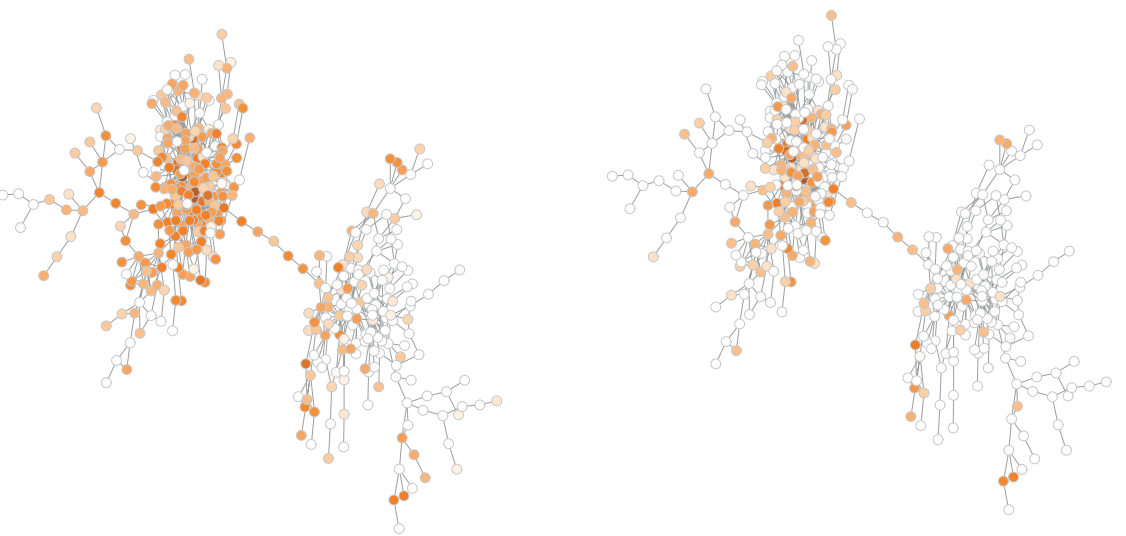


\section{Supplementary Figure 8:}

A
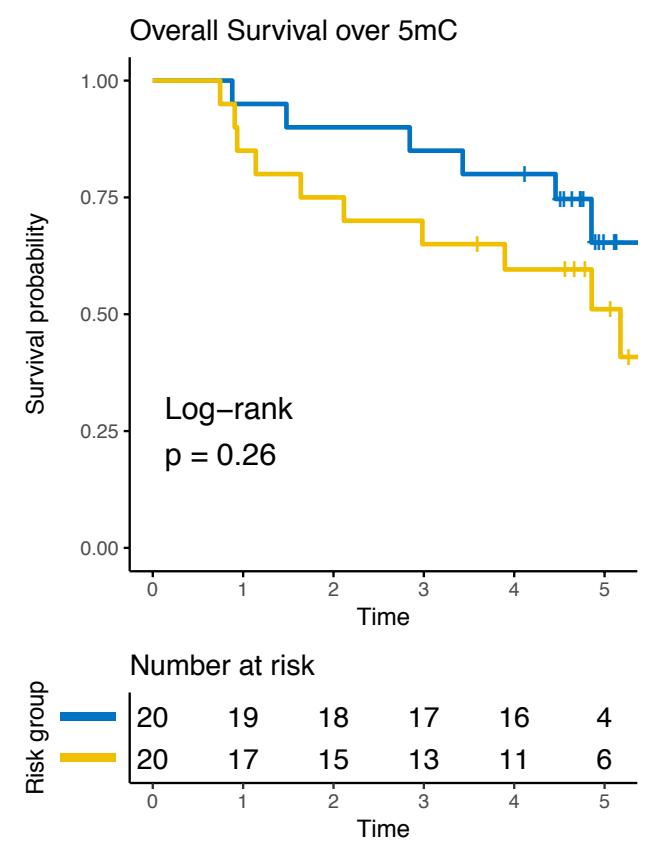

C
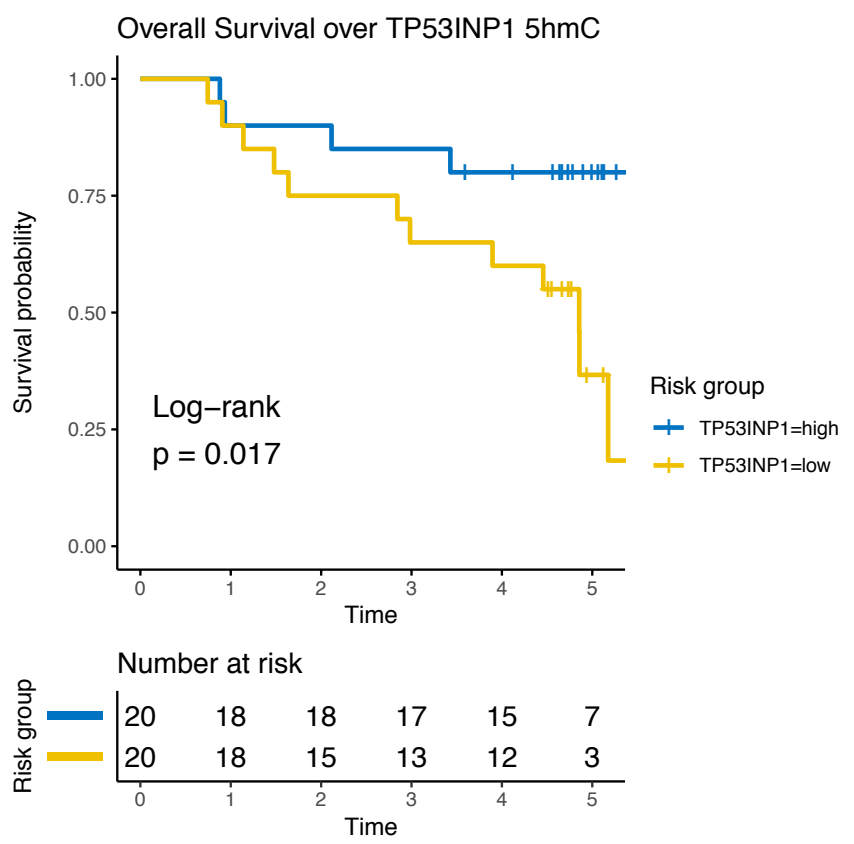

B

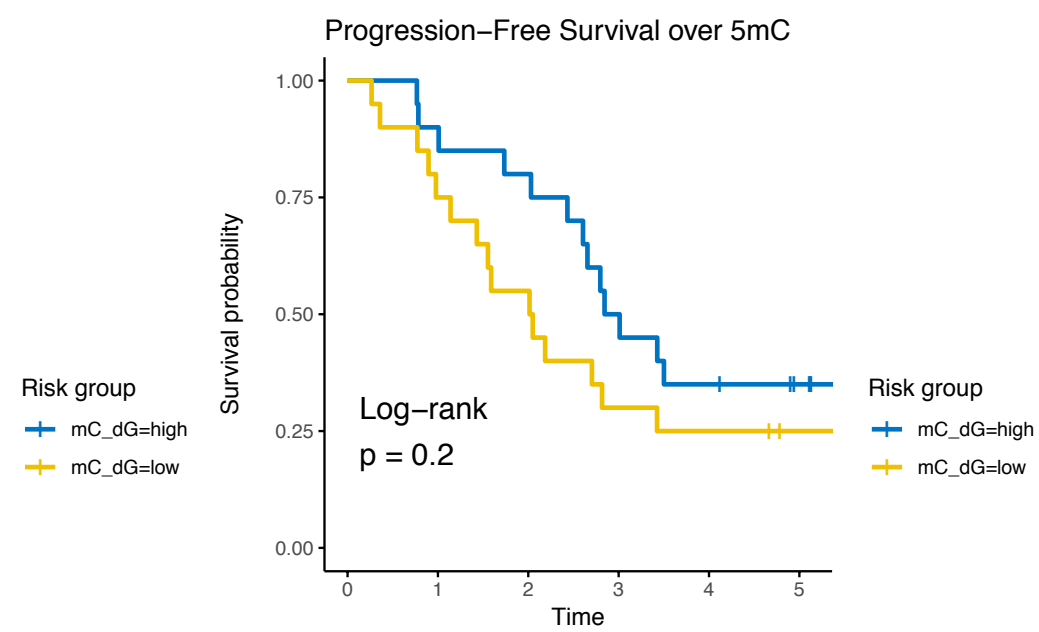

D

Overall Survival over Gene Expression TP53INP1-UPF1-RNF144A

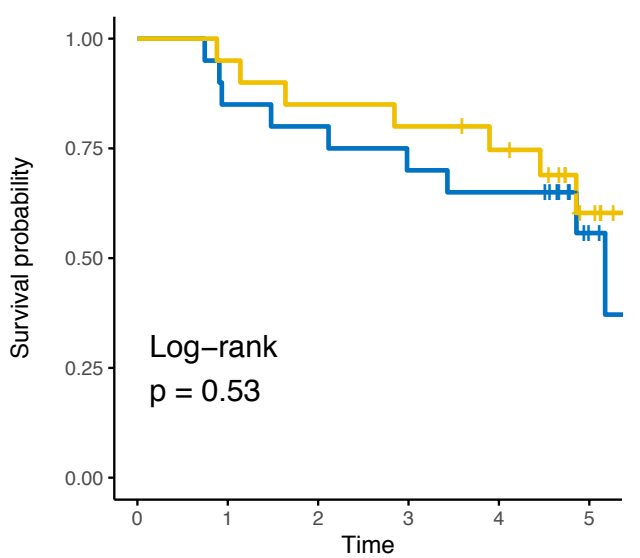

Risk group

+ sig.expr=high + sig.expr=low 